\title{
Modèles mathématiques de digestion anaérobie: effet de l'hydrolyse sur la production du biogaz
}

\author{
Y. Daoud a,b,* , N. Abdellatif a,c, J. Harmand d \\ a Université de Tunis El Manar, ENIT, LAMSIN, BP 37, Le Belvédère, 1002 Tunis, Tunisie. \\ b UMR Mistea, Université Montpellier, INRA, 34060 Montpellier, France. \\ (E-mail: daoud_yessmine@yahoo.fr) \\ c Université de Manouba, ENSI, Campus Universitaire de Manouba, 2010 Manouba, Tunisie. \\ (E-mail: nahla.abdellatif@ensi-uma.tn) \\ d LBE, Université Montpellier, INRA, Narbonne, France. (E-mail: jerome.harmand@inra.fr) \\ * Corresponding author.
}

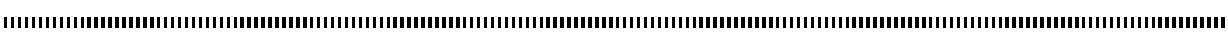

RÉSUMÉ. Nous analysons, dans ce travail, l'effet de l'hydrolyse sur le comportement du processus de digestion anaérobie et sur la production du biogas (méthane et hydrogène). Nous considérons deux modélisations possibles de l'hydrolyse. Nous supposons, dans un premier temps, que l'hydrolyse se fait d'une manière purement enzymatique puis nous faisons intervenir un compartiment microbien hydrolytique dans le modèle. Les modèles considérés font intervenir l'inhibition de la croissance des bactéries acétogènes (respectivement méthanogènes hydrogénotrophes) par l'hydrogène (respectivement, par l'acétate). Pour analyser l'effet de ces inhibitions, en présence de l'étape d'hydrolyse, nous faisons, dans un premier temps, l'étude d'un modèle sans inhibition. Nous déterminons les équilibres des modèles étudiés et nous donnons des conditions nécessaires et suffisantes pour leur stabilité. Les régions d'existence et de stabilité des équilibres sont illustrées par des diagrammes opératoires. Nous montrons que la modélisation de la phase d'hydrolyse par une activité enzymatique constante affecte la production du méthane et de l'hydrogène. En outre, l'introduction du compartiment microbien hydrolytique fait apparaître de nouveaux équilibres et affecte les régions de stabilité. Nous prouvons que le taux maximal de méthane et d'hydrogène produit est atteint en un équilibre stable.

ABSTRACT. We investigate, in this work, the effects of hydrolysis on the behavior of the anaerobic digestion process and the production of biogas (namely, the methane and the hydrogen). Two modelisations of the hydrolysis process are involved. We consider, first, that the microbial enzymatic activity is constant, then we take into consideration an explicit hydrolytic microbial compartiment for the substrate biodegradation. The considered models include the inhibition of acetoclastic and hydrogenotrophic methanogens. To examine the effects of these inhibitions in presence of a hydrolysis step, we first study an inhibition-free model. We determine the steady states and give sufficient and necessary conditions for their stability. The existence and stability of the steady states are illustrated by operating diagrams. We prove that modelling the hydrolysis phase by a constant enzymatic activity affects the production of methane and hydrogen. Furthermore, introducing the hydrolytic microbial compartment makes appear new steady states and affects the stability regions. We prove that the maximal rate production of methane and hydrogen is reached in one stable steady-state.

MOTS-CLÉS : Digestion anaérobie, chemostat, stabilité, diagrammes opératoires, production biogaz.

KEYWORDS : Anaerobic digestion, chemostat, stability, operating diagrams, biogas production.

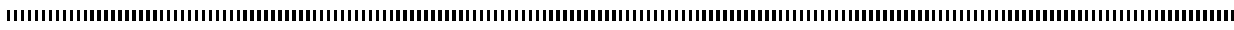




\section{Introduction}

La digestion anaérobie (D.A.) est un processus naturel au cours duquel la matière organique se transforme en biogaz dans un milieu sans oxygène. Elle est utilisée pour le traitement des eaux usées et des déchets organiques solides pour la production de méthane ou d'hydrogène. La digestion anaérobie peut-être décrite synthétiquement comme un processus comprenant quatre grandes étapes : l'hydrolyse, l'acidogenèse, l'acétogenèse, et la méthanogenèse hydrogénétrophe et acétoclastique. Au cours de la première étape, les molécules organiques complexes $\left(X_{0}\right)$ sont décomposées en substrat simple $(S)$. Pendant l'acidogenèse, les bactéries acidogènes $\left(X_{S}\right)$ convertissent le substrat $(S)$ en acide acétique $(A)$, acide gras volatile $(V)$ et alcool, hydrogène $(H)$ et dioxyde de carbone. Ensuite, l'acide gras volatile et les alcools sont consommés par les bactéries acétogènes $\left(X_{V}\right)$ et ils sont convertis en acide acétique $(A)$ ainsi qu'en dioxyde de carbone et hydrogène $(H)$. Dans la phase finale, les bactéries méthanogènes acétoclastes $\left(X_{A}\right)$ convertissent l'acide acétique $(A)$ en méthane et en dioxyde de carbone, tandis que les bactéries méthanogènes hydrogénotrophes $\left(X_{H}\right)$ convertissent l'hydrogène $(H)$ et le dioxyde de carbone en méthane, voir Figure 1.

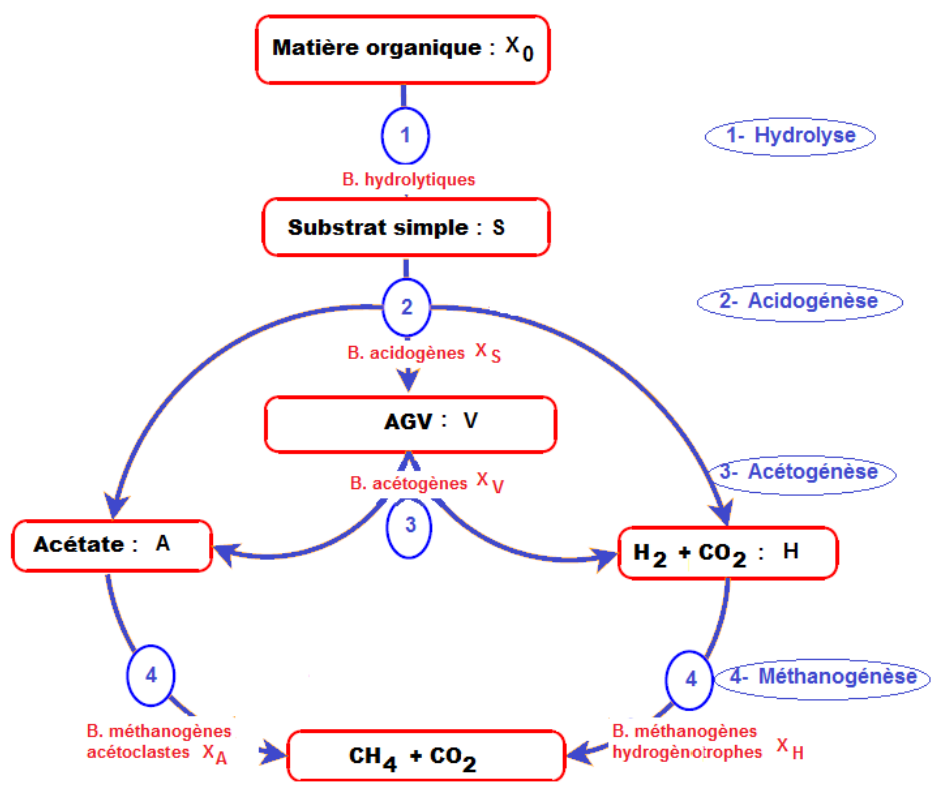

Figure 1. Les étapes de la digestion anaérobie

La majorité des études faites sur les modèles de digestion anérobie s'intéressent aux trois principales phases : l'acidogenèse, l'acétogenèse et la méthanogenèse. Néanmoins, il a été prouvé que l'activité des bactéries hydrolytiques, durant l'étape d'hydrolyse, peut affecter l'ensemble du système, voir [7], [10]. Cette étape permet la dégradation des substrats sous forme solide (matière organique) en substrats sous forme soluble (substrat 
simple). Ce processus d'hydrolyse peut-être modélisé de manières différentes :

- on peut considérer que l'hydrolyse se fait d'une manière purement enzymatique.

- ou on tient compte des bactéries qui dégradent la matière organique pour produire du substrat simple, le modèle est alors dit avec compartiment microbien hydrolytique.

L'étude d'un modèle de digestion anaérobie à trois étapes incluant une phase d'hydrolyse a été faite dans [2]. Il a été démontré que le système peut avoir des comportements qualitatifs très différents, selon la modélisation de l'hydrolyse considérée et qu'il peut présenter, selon les conditions initiales, de la bistabilité ou de la quadri-stabilité.

Nous nous intéressons aux modèles décrits dans [11] où trois des quatre étapes principales de digestion anaérobie, l'acidogenèse, l'acétogenèse et la méthanogenèse, sont décrites par un système d'équations différentielles, modélisant l'interaction des populations microbiennes dans un chemostat. Les micro-organismes consomment et/ou produisent des substrats simples, des alcools et des acides gras, de l'acide acétique et de l'hydrogène. Les bactéries acétogènes et méthanogènes hydrogénotrophes interagissent par une relation de syntrophie. Le modèle comprend également l'inhibition des bactéries méthanogènes acétoclastes et hydrogénotrophes. La première étape de digestion anaérobie qui est l'étape d'hydrolyse n'est pas modélisée dans [11]. Nous nous proposons d'ajouter cette étape d'hydrolyse et d'étudier les systèmes résultants. Nous déterminons les équilibres et nous étudions leur stabilité. Nous nous intéressons également au taux de biogas produit. Les modèles que nous nous proposons d'étudier s'écrivent sous la forme :

$$
\left\{\begin{array}{l}
\frac{d X_{0}}{d t}=D\left(X_{0 i n}-X_{0}\right)-r_{0} \\
\frac{d S}{d t}=D\left(S_{i n}-S\right)-\frac{1}{c_{s}} g_{S}(S) X_{S}+k_{0} r_{0} \\
\frac{d X_{S}}{d t}=\left(g_{S}(S)-D\right) X_{S} \\
\frac{d V}{d t}=-D V+\gamma_{s v} g_{S}(S) X_{S}-\frac{1}{c_{v}} g_{V}(V, H) X_{V} \\
\frac{d X_{V}}{d t}=\left(g_{V}(V, H)-D\right) X_{V} \\
\frac{d A}{d t}=-D A+\gamma_{s a} g_{S}(S) X_{S}+\gamma_{v a} g_{V}(V, H) X_{V}-\frac{1}{c_{a}} g_{A}(A) X_{A} \\
\frac{d X_{A}}{d t}=\left(g_{A}(A)-D\right) X_{A} \\
\frac{d H}{d t}=-D H+\gamma_{s h} g_{S}(S) X_{S}+\gamma_{v h} g_{V}(V, H) X_{V}-\frac{1}{c_{h}} g_{H}(H, A) X_{H} \\
\frac{d X_{H}}{d t}=\left(g_{H}(H, A)-D\right) X_{H} .
\end{array}\right.
$$

$X_{0 i n}$ est la concentration du substrat solide (lentement biodégradable) à l'entrée du chémostat. $r_{0}$ est la vitesse de réaction de l'hydrolyse qui dépend des variables d'état selon la modélisation considérée. $S_{i n}$ est la concentration du substrat soluble (rapidement biodégradable) à l'entrée du chémostat et $D$ est le taux de dilution. $c_{s}, c_{v}, c_{a}, c_{h}$ sont les coefficients de rendement des bactéries et $\gamma_{s v}, \gamma_{s a}, \gamma_{s h}, \gamma_{v a}, \gamma_{v h}$ sont des rapports entre le rendement du produit et le rendement de la biomasse. $g_{S}(),. g_{A}(),. g_{V}(.,$.$) et g_{H}(.,$. 
sont les taux de croissance spécifiques. Nous supposons dans toute la suite qu'ils sont de classe $\mathcal{C}^{1}$.

Plusieurs facteurs sont susceptibles d'inhiber cette croissance. Dans le modèle (1), les inhibitions suivantes seront intégrées :

- l'inhibition des bactéries acétogènes par un excès d'hydrogène dans le système.

- l'inhibition des bactéries méthanogènes hydrogénotrophes par des concentrations élevées de l'acide acétique $A$

- la croissance des bactéries méthanogènes acétoclastes n'a lieu que pour certaines concentrations de l'acide acétique $A$.

L'objectif de ce travail est d'étudier, dans un premier temps, l'effet de l'hydrolyse sur les propriétés d'un modèle sans inhibition, puis d'un modèle tenant compte des inhibitions. L'hydrolyse est modélisée des deux manières décrites précédemment. Hormis la détermination des équilibres et l'analyse de la stabilité, nous nous proposons de déterminer laquelle des voies métaboliques du processus de la digestion anaérobie permet de maximiser les taux de biogas (méthane et hydrogène) produits, dans des modèles incluant l'étape d'hydrolyse. L'optimisation du biogaz a été considérée en particulier dans [12] et plus récemment dans [1], pour des modèles à deux ou à trois étapes. Dans ce travail, l'étude combine et étend les résultats obtenus dans [2] pour un modèle à deux étapes et ceux de [11], qui ne tient pas compte de l'étape de l'hydrolyse.

Cet article est organisé comme suit. Dans la section 2, nous analysons le modèle (1) où l'hydrolyse est considérée comme une phase purement enzymatique. La vitesse de la réaction est donnée par $r_{0}(t)=k_{h y d} X_{0}(t)$, où $k_{h y d}$ est une constante et $X_{0}$ est la concentration du substrat lentement biodégradable. Pour étudier l'effet de l'inhibition en présence de la phase d'hydrolyse, nous considérons, dans la section 2.1, le modèle (1) sans tenir compte des inhibitions décrites précédemment. Nous déterminons les équilibres du système et nous étudions leur stabilité globale. Ces résultats sont illustrés par des diagrammes opératoires qui décrivent les régions d'existence et de stabilité des équilibres, en fonction des paramètres de contrôle $D, S_{i n}$ et $X_{0 i n}$. Nous nous intéressons également aux taux maximaux du méthane et de l'hydrogène produits. Ce taux est exprimé en fonction de la dilution $D$, pour des valeurs des concentrations $X_{0 i n}$ et $S_{i n}$ fixés dans un intervalle de valeurs donné. Dans la section 2.2, les inhibitions sont intégrées dans le modèle. L'analyse des équilibres est effectuée. Les régions de stabilité des équilibres sont illustrées par des diagrammes opératoires et les taux de méthane et d'hydrogène produits sont déterminés. Dans la section 3, nous étudions le modèle (1) en considérant un compartiment microbien hydrolytique pour modéliser la phase d'hydrolyse. La vitesse de réaction est alors $r_{0}(t)=g_{0}\left(X_{0}(t)\right) X_{S}(t)$, avec $g_{0}($.$) est le taux de croissance microbienne spéci-$ fique de $X_{S}$ sur $X_{0}$. L'étude du sous-modèle composé des trois premières équations du modèle (1) montre l'existence d'au maximum trois équilibres, ce qui donne naissance au maximum dix-sept équilibres du système complet (1). Vu la complexité de l'étude dans ce cas, nous nous limitons au cas sans inhibition. L'existence et la stabilité des équilibres sont discutées et les résultats obtenus sont illustrés par des diagrammes opératoires dans le plan $\left(D, S_{i n}\right)$. Nous montrons que la production maximale du méthane et de l'hydrogène varie suivant les concentrations des substrats lentement et rapidement biodégradable à l'entrée. 


\section{L'analyse du modèle avec hydrolyse enzymatique}

On considère, ici, le modèle (1) avec $r_{0}=k_{h y d} X_{0}$. Nous commençons par étudier le modèle sans effet d'inhibition. Cela veut dire que la fonction de croissance $g_{V}$ ne dépend donc pas de $H$, que $g_{H}$ ne dépend pas de $A$ et que $g_{A}$ est une fonction croissante en $A$. Nous généralisons, ensuite, notre étude au cas de l'inhibition par l'acétate et par l'hydrogène.

\subsection{Le modèle sans inhibition}

Dans ce cas, on pose $g_{V}(V)=g_{V}(V, 0)$ et $g_{H}(H)=g_{H}(H, 0)$ et on suppose que :

(H1) $g_{S}(0)=0$ et $g_{S}($.$\left.) est croissante sur \right] 0,+\infty[$.

(H2) $g_{V}(0)=0$ et $g_{V}($.$\left.) est croissante sur \right] 0,+\infty[$.

(H3) $g_{A}(0)=0$ et $g_{A}($.$\left.) est croissante sur \right] 0,+\infty[$.

(H4) $g_{H}(0)=0$ et $g_{H}($.$\left.) est croissante sur \right] 0,+\infty[$.

Pour $l=S, V, A$ et $H$, on note $\lambda_{l}$ la solution de l'équation $g_{l}\left(\lambda_{l}\right)=D$, si elle existe et $\lambda_{l}=+\infty$, sinon.

Notons que, d'après le principe de la conservation de la matière, on a

$$
1+\gamma_{s v}+\gamma_{s a}+\gamma_{s h} \leqslant \frac{1}{c_{s}}
$$

C'est à dire que la quantité de $S$ dégradée est supérieure ou égale à la somme des quantités de $X_{S}$ qui s'est développée et de $V, A$ et $H$ produites. De la même manière, on a $1+\gamma_{v a}+$ $\gamma_{v h} \leqslant \frac{1}{c_{v}}, 1 \leqslant \frac{1}{c_{a}}$ et $1 \leqslant \frac{1}{c_{h}}$.

Nous commençons par montrer le résultat suivant :

Proposition 2.1. Pour des valeurs initiales positives, les solutions du système (1) restent positives et bornées, pour tout $t \geq 0$.

Preuve : On peut montrer par des arguments standards la positivité des solutions de (1), voir par exemple [2]. Pour démontrer que toutes les solutions de (1) sont bornées, on pose

$$
\begin{gathered}
Z=k_{0} X_{0}+S+\frac{1}{c_{s}} X_{S}+V+\frac{1}{c_{v}} X_{V}-\gamma_{s v} X_{S}+A+\frac{1}{c_{a}} X_{A}-\gamma_{v a} X_{V}-\gamma_{s a} X_{S} \\
+H+\frac{1}{c_{h}} X_{H}-\gamma_{v h} X_{V}-\gamma_{s h} X_{S} .
\end{gathered}
$$

En dérivant $Z$, on montre que $Z^{\prime}=D\left(S_{i n}^{+}-Z\right)$, avec $S_{i n}^{+}=k_{0} X_{0 i n}+S_{i n}$. On pose alors $V=Z-S_{i n}^{+}$. En appliquant le lemme de Gronwall, on obtient $V(t)=V(0) e^{-D t}$, pour tout $t \geqslant 0$. Par conséquent,

$$
Z(t)=S_{\text {in }}^{+}+\left(Z(0)-S_{\text {in }}^{+}\right) e^{-D t}, \text { pour tout } t \geqslant 0
$$

et on en déduit que

$$
Z(t) \leqslant \max \left(Z(0), k_{0} X_{0 i n}+S_{\text {in }}\right), \text { pour tout } t \geqslant 0 .
$$

Comme $Z=k_{0} X_{0}+S+\left(\frac{1}{c_{s}}-\gamma_{s v}-\gamma_{s a}-\gamma_{s h}\right) X_{S}+V+\left(\frac{1}{c_{v}}-\gamma_{v a}-\gamma_{v h}\right) X_{V}+A+$ $\frac{1}{c_{a}} X_{A}+H+\frac{1}{c_{h}} X_{H}$. D'après la remarque précédente, on a $\frac{1}{c_{s}}-\gamma_{s v}-\gamma_{s a}-\gamma_{s h}>0$ et $\frac{1}{c_{v}}-\gamma_{v a}-\gamma_{v h}>0$. Par suite, on peut conclure que les solutions de (1) sont bornées, pour tout $t \geqslant 0$. 


\subsubsection{Existence et stabilité des équilibres}

La première équation du modèle (1) peut être découplée du reste du système. En effet, l'évolution de la variable $X_{0}$ est indépendante de l'évolution des autres variables. L'étude de la première équation du modèle (1) montre qu'à l'équilibre, $X_{0}$ converge globalement vers $X_{0}^{*}=\frac{D}{D+k_{h y d}} X_{0 i n}>0$.

De même, l'évolution du couple $\left(S, X_{S}\right)$ est indépendante de l'évolution des variables $V, A$ et $H$. A l'équilibre de $X_{0}$, la deuxième et la troisième équation de (1) peuvent être découplées des équations suivantes, elles se réduisent aux équations d'un chémostat simple. On peut en déduire que si $S_{i n}^{*}=S_{i n}+\frac{k_{0} k_{h y d}}{D} X_{0}^{*}<\lambda_{S}$ alors $\left(S_{i n}^{*}, 0\right)$ est le seul équilibre dans $[0,+\infty[\times[0,+\infty[$. Il est globalement asymptotiquement stable. Si $S_{i n}^{*}>\lambda_{S}$ alors l'équilibre $\left(\lambda_{S}, X_{S}^{*}\right)$ existe dans $] 0,+\infty[\times] 0,+\infty[$. Il est globalement asymptotiquement stable et l'équilibre $\left(S_{i n}^{*}, 0\right)$ est instable, voir [9].

D’après ce qui précède, on peut déduire que le système (1) possède neuf équilibres donnés dans la Proposition 2.2.

Proposition 2.2. Sous les hypothèses (H1)-(H4), le système (1) possède neuf points d'équilibre décrits dans le tableau 1.

\begin{tabular}{|c||c|c|c|c|c|c|c|c|c|}
\hline Équilibre & $X_{0}$ & $S$ & $X_{S}$ & $V$ & $X_{V}$ & $A$ & $X_{A}$ & $H$ & $X_{H}$ \\
\hline \hline$E_{l}$ & $X_{0}^{*}$ & $S_{i n}^{*}$ & 0 & 0 & 0 & 0 & 0 & 0 & 0 \\
\hline$E_{0}$ & $X_{0}^{*}$ & $\lambda_{S}$ & $X_{S}^{*}$ & $V^{(0)}$ & 0 & $A^{(0)}$ & 0 & $H^{(0)}$ & 0 \\
\hline$E_{H}$ & $X_{0}^{*}$ & $\lambda_{S}$ & $X_{S}^{*}$ & $V^{(0)}$ & 0 & $A^{(0)}$ & 0 & $\lambda_{H}$ & $\begin{array}{c}c_{h}\left(H^{(0)}\right. \\
\left.-\lambda_{H}\right)\end{array}$ \\
\hline$E_{A}$ & $X_{0}^{*}$ & $\lambda_{S}$ & $X_{S}^{*}$ & $V^{(0)}$ & 0 & $\lambda_{A}$ & $c_{a}\left(A^{(0)}-\lambda_{A}\right)$ & $H^{(0)}$ & 0 \\
\hline$E_{A H}$ & $X_{0}^{*}$ & $\lambda_{S}$ & $X_{S}^{*}$ & $V^{(0)}$ & 0 & $\lambda_{A}$ & $c_{a}\left(A^{(0)}-\lambda_{A}\right)$ & $\lambda_{H}$ & $\begin{array}{c}c_{h}\left(H^{(0)}\right. \\
\left.-\lambda_{H}\right)\end{array}$ \\
\hline$E_{V}$ & $X_{0}^{*}$ & $\lambda_{S}$ & $X_{S}^{*}$ & $\lambda_{V}$ & $X_{V}^{*}$ & $\bar{A}$ & 0 & $\bar{H}$ & 0 \\
\hline$E_{V H}$ & $X_{0}^{*}$ & $\lambda_{S}$ & $X_{S}^{*}$ & $\lambda_{V}$ & $X_{V}^{*}$ & $\bar{A}$ & 0 & $\lambda_{H}$ & $\begin{array}{c}c_{h}(\bar{H} \\
\left.-\lambda_{H}\right)\end{array}$ \\
\hline$E_{V A}$ & $X_{0}^{*}$ & $\lambda_{S}$ & $X_{S}^{*}$ & $\lambda_{V}$ & $X_{V}^{*}$ & $\lambda_{A}$ & $c_{a}\left(\bar{A}-\lambda_{A}\right)$ & $\bar{H}$ & 0 \\
\hline$E_{*}$ & $X_{0}^{*}$ & $\lambda_{S}$ & $X_{S}^{*}$ & $\lambda_{V}$ & $X_{V}^{*}$ & $\lambda_{A}$ & $c_{a}\left(\bar{A}-\lambda_{A}\right)$ & $\lambda_{H}$ & $c_{h}(\bar{H}$ \\
& & & & & & & & & $\left.-\lambda_{H}\right)$ \\
\hline
\end{tabular}

Tableau 1. Les équilibres du modèle (1) sans inhibition, dans le cas où $r_{0}=k_{h y d} X_{0}$

avec

- $X_{0}^{*}=\left(\frac{D}{D+k_{h y d}}\right) X_{0 i n}, S_{i n}^{*}=\left(\frac{k_{0} k_{h y d}}{D+k_{h y d}}\right) X_{0 i n}+S_{i n}$,

- $X_{S}^{*}=c_{s}\left(S_{i n}^{*}-\lambda_{S}\right), X_{V}^{*}=c_{v}\left(V^{(0)}-\lambda_{V}\right)$,

- $V^{(0)}=\gamma_{s v} X_{S}^{*}, A^{(0)}=\gamma_{s a} X_{S}^{*}, H^{(0)}=\gamma_{s h} X_{S}^{*}$,

- $\bar{A}=A^{(0)}+\gamma_{v a} X_{V}^{*}, \bar{H}=H^{(0)}+\gamma_{v h} X_{V}^{*}$.

Notons qu'en ajoutant l'étape d'hydrolyse enzymatique dans le modèle étudié dans [11], une nouvelle composante $X_{0}^{*}$ apparaît dans les composantes de chaque équilibre

et le substrat à l'entrée $S_{i n}$ devient plus grand $S_{i n}^{*}=\left(\frac{k_{0} k_{h y d}}{D+k_{h y d}}\right) X_{0 i n}+S_{i n}$. Ainsi, la concentration des bactéries acidogènes $X_{S}^{*}$ augmente quand $S_{i n}$ augmente. De même, pour les bactéries $X_{V}, X_{A}$ et $X_{H}$ et les concentrations de $V, A$ et $H$. La condition de 
persistance de $X_{S}$ pour le modèle sans hydrolyse est $S_{i n}>\lambda_{S}$ et pour le modèle avec hydrolyse est $S_{i n}^{*}>\lambda_{S}$, avec $S_{i n}^{*}=\left(\frac{k_{0} k_{h y d}}{D+k_{h y d}}\right) X_{0 i n}+S_{i n}>S_{i n} . X_{S}$ se trouve donc favorisé par l'ajout du terme d'hydrolyse, puisque recevant davantage de substrat. En appliquant la même technique que [11, Lemme 3.1], on peut déduire un résultat de stabilité globale des équilibres du système (1). Les conditions d'existence et de stabilité globale des équilibres du système (1) sont résumées dans la proposition suivante :

Proposition 2.3. Sous les hypothèses $(\mathbf{H 1})$ - $(\mathbf{H 4})$, les conditions d'existence et de stabilité des équilibres du système (1) sont données par:

\begin{tabular}{|c||c|c|}
\hline L'équilibre & Conditions d'existence & Conditions de stabilité globale \\
\hline \hline$E_{l}$ & toujours & $S_{i n}^{*}<\lambda_{S}$ \\
\hline$E_{0}$ & $S_{i n}^{*}>\lambda_{S}$ & $A^{(0)}<\lambda_{A}, H^{(0)}<\lambda_{H}$ \\
& et $V^{(0)}<\lambda_{V}$ \\
\hline$E_{H}$ & $H^{(0)}>\lambda_{H}$ & $A^{(0)}<\lambda_{A}$ et $V^{(0)}<\lambda_{V}$ \\
\hline$E_{A}$ & $A^{(0)}>\lambda_{A}$ & $H^{(0)}<\lambda_{H}$ et $V^{(0)}<\lambda_{V}$ \\
\hline$E_{A H}$ & $A^{(0)}>\lambda_{A}$ et $H^{(0)}>\lambda_{H}$ & $V^{(0)}<\lambda_{V}$ \\
\hline$E_{V}$ & $V^{(0)}>\lambda_{V}$ & $\bar{A}<\lambda_{A}$ et $\bar{H}<\lambda_{H}$ \\
\hline$E_{V H}$ & $V^{(0)}>\lambda_{V}$ et $\bar{H}>\lambda_{H}$ & $\bar{A}<\lambda_{A}$ \\
\hline$E_{V A}$ & $V^{(0)}>\lambda_{V}$ et $\bar{A}>\lambda_{A}$ & $\bar{H}<\lambda_{H}$ \\
\hline$E_{*}$ & $V^{(0)}>\lambda_{V}, \bar{A}>\lambda_{A}$ et $\bar{H}>\lambda_{H}$ & Lorsqu'il existe \\
\hline
\end{tabular}

Tableau 2. Les conditions d'existence et de stabilité des équilibres du système (1) sans inhibition, dans le cas où $r_{0}=k_{\text {hyd }} X_{0}$.

La preuve de la proposition 2.3 et des propositions suivantes sont données dans l'annexe.

\subsubsection{Diagrammes opératoires}

Les diagrammes opératoires décrivent, dans le plan, les régions de stabilité des équilibres selon les paramètres de contrôle $D, S_{i n}$ et $X_{0 i n}$. Dans un premier temps, $X_{0 i n}$ est fixé et le comportement du système (1) est décrit dans le plan $\left(D, S_{i n}\right)$. Ensuite, nous fixons la valeur de $S_{i n}$ et nous traçons les diagrammes opératoires dans le plan $\left(D, X_{0 i n}\right)$.

\subsubsection{Diagrammes opératoires dans le plan $\left(D, S_{i n}\right)$}

Nous traçons les diagrammes opératoires dans le plan $\left(D, S_{i n}\right)$ pour des valeurs différentes de la concentration $X_{0 i n}$ du substrat solide à l'entrée. Ces diagrammes sont obtenus en prenant des fonctions de croissance de type Monod :

$$
g_{l}(l)=\frac{m_{l} l}{k_{l}+l}, \quad \text { avec } \quad l=S, V, A, H .
$$

Les valeurs des paramètres $m_{l}$ et $k_{l}, l=S, V, A, H$, sont celles du tableau A1 de [11]. Ces fonctions vérifient les hypothèses $(\mathbf{H 1})-(\mathbf{H} 4)$. Les régions $R_{1}, \cdots, R_{5}$ sont définies dans la proposition 2.4 et correspondent, respectivement, aux régions d'existence et de stabilité des équilibres $E_{l}, E_{0}, E_{H}, E_{A H}$ et $E_{*}$. 
La figure 2 représente les diagrammes opératoires dans le plan $\left(D, S_{i n}\right)$ pour $X_{0 i n}=$ 0 et $X_{0 i n}=1$. Pour $X_{0 i n}=0$, on obtient le même diagramme opératoire de [11] (voir Figure 4 de [11]).
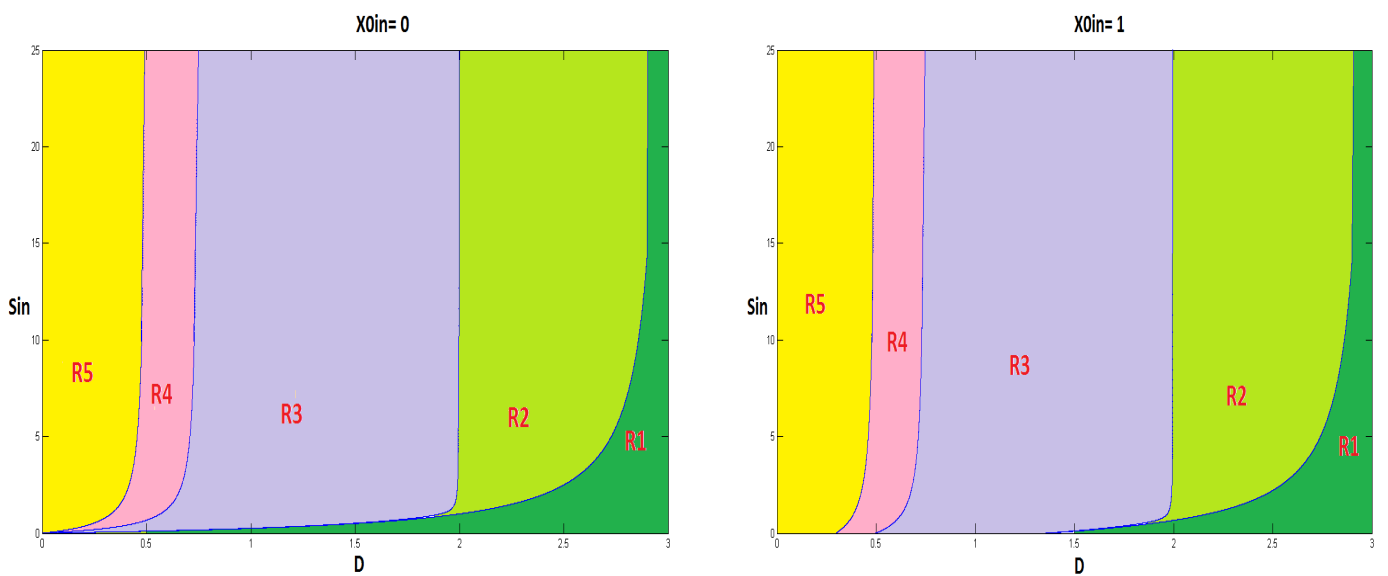

Figure 2. Diagramme opératoire, dans le plan $\left(D, S_{i n}\right)$, à gauche pour $X_{0 i n}=0$, à droite pour $X_{0 \text { in }}=1$.

Notons pour $l=S, V, A$ et $H$, les solutions respectives $\lambda_{l}$ des équations $g_{l}\left(\lambda_{l}\right)=D$. Elles sont données par:

$$
\lambda_{S}=\frac{D k_{s}}{m_{s}-D}, \quad \lambda_{V}=\frac{D k_{v}}{m_{v}-D}, \quad \lambda_{A}=\frac{D k_{a}}{m_{a}-D}, \quad \text { et } \quad \lambda_{H}=\frac{D k_{h}}{m_{h}-D} .
$$

Elles sont définies pour $0<D<\min \left(m_{s}, m_{v}, m_{a}, m_{h}\right)$.

Définissons les fonctions $F, K_{H}, K_{A}$ et $K_{V}$ par:

$$
F(D)=\lambda_{S}-\frac{k_{0} k_{h y d}}{D+k_{h y d}} X_{0 i n}, \quad K_{H}(D)=\frac{\lambda_{H}}{\gamma_{s h} c_{s}}, \quad K_{A}(D)=\frac{\lambda_{A}}{\gamma_{s a} c_{s}} \text { et } K_{V}(D)=\frac{\lambda_{V}}{\gamma_{s v} c_{s}} \text {. }
$$

Nous nous plaçons dans le cas où :

$$
g_{V}\left(V^{(0)}\right)<g_{A}(\bar{A})<g_{H}(\bar{H})<g_{A}\left(A^{(0)}\right)<g_{H}\left(H^{(0)}\right)<g_{S}\left(S_{i n}^{*}\right) .
$$

Ces conditions sont vérifiées par le jeu de paramètres choisi dans [11], voir tableau A1. Si l'inégalité (3) est vérifiée, on peut montrer en utilisant la proposition 2.3 que :

Proposition 2.4. Le plan $\left(D, S_{i n}\right)$ est divisé en cinq régions décrites dans le tableau 3 : 


\begin{tabular}{|c||c||c|c|c|c|c|c|c|c|c|}
\hline Condition & Région & $E_{l}$ & $E_{0}$ & $E_{H}$ & $E_{A}$ & $E_{A H}$ & $E_{V}$ & $E_{V H}$ & $E_{V A}$ & $E_{*}$ \\
\hline \hline$S_{i n}<F(D)$ & $R_{1}$ & GAS & & & & & & & & \\
\hline $\begin{array}{c}S_{i n}>F(D) \\
\text { et } S_{i n}<K_{H}(D)+F(D)\end{array}$ & $R_{2}$ & I & GAS & & & & & & & \\
\hline $\begin{array}{c}S_{i n}>K_{H}(D)+F(D) \\
\text { et } S_{i n}<K_{A}(D)+F(D)\end{array}$ & $R_{3}$ & I & I & GAS & & & & & & \\
\hline $\begin{array}{c}S_{i n}>K_{A}(D)+F(D) \\
\text { et } S_{i n}<K_{V}(D)+F(D)\end{array}$ & $R_{4}$ & I & I & I & I & GAS & & & & \\
\hline$S_{i n}>K_{V}(D)+F(D)$ & $R_{5}$ & I & I & I & I & I & I & I & I & GAS \\
\hline
\end{tabular}

Tableau 3. Existence et stabilité des équilibres du système (1) sans inhibition, dans le cas où $r_{0}=k_{\text {hyd }} X_{0}$, selon $\left(D, S_{\text {in }}\right)$

L'abréviation GAS (resp. I) signifie que l'équilibre correspondant est globalement asymptotiquement stable (resp. instable). L'absence de lettres signifie que l'équilibre n'existe pas.

En augmentant la valeur du substrat solide $X_{0 i n}$, on peut remarquer que la taille des régions de stabilité s'agrandit. Par suite, en ajoutant l'étape d'hydrolyse, les régions de stabilité des équilibres dans le plan $\left(D, S_{i n}\right)$ augmentent de taille en fonction de la concentration $X_{0 i n}$ du substrat solide à l'entrée. D'après la Figure 2 à droite, quand la concentration du substrat solide $X_{0 i n}$ devient plus grande et que le taux de dilution $D$ est faible (région $R_{5}$ ) alors pour n'importe quelle valeur de substrat soluble $S_{i n}$, le système converge vers l'équilibre de coexistence $E^{*}$. Par contre, si le taux de dilution $D$ est important (région $R_{1}$ ), le système converge vers l'équilibre de lessivage $E_{l}$.

Les équilibres $E_{A}, E_{V}, E_{V H}$ et $E_{V A}$ sont instables, dans ce cas.

\subsubsection{Diagrammes opératoires dans le plan $\left(D, X_{0 i n}\right)$}

Nous traçons le diagramme opératoire dans le plan $\left(D, X_{0 i n}\right)$ pour différentes valeurs de $S_{i n}$. La Figure 3 présente les diagrammes opératoires dans le plan $\left(D, X_{0 i n}\right)$ pour $S_{i n}=0$ et $S_{i n}=1$. Les régions $J_{1}, \cdots, J_{5}$ sont définies dans la proposition 2.5 et correspondent, respectivement, aux régions d'existence et de stabilité des équilibres $E_{l}, E_{0}, E_{H}, E_{A H}$ et $E_{*}$.
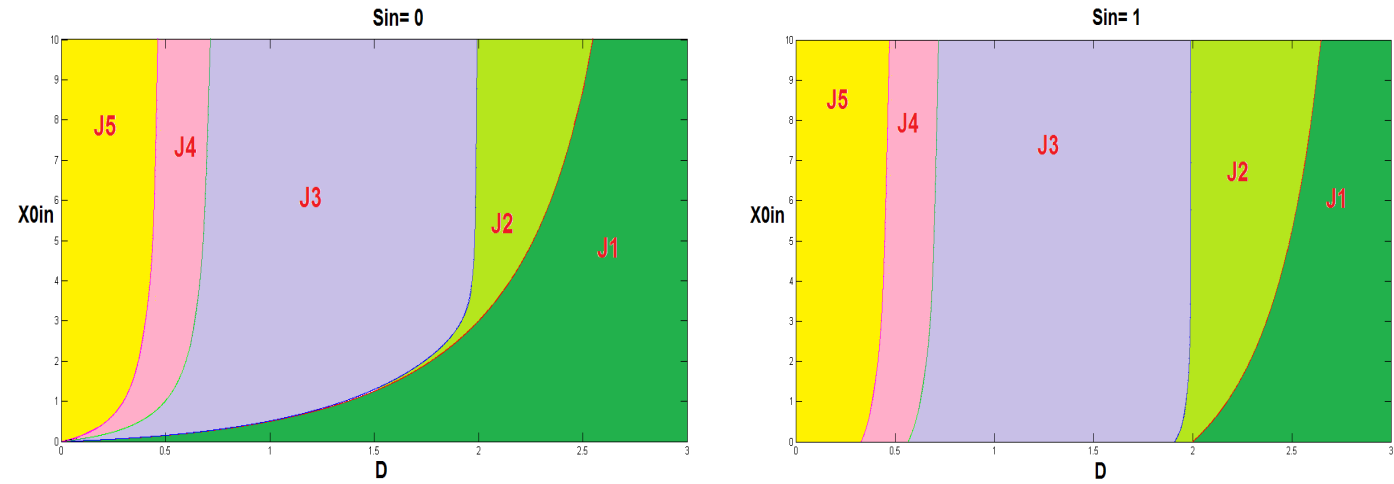

Figure 3. Diagrammes opératoires dans le plan $\left(D, X_{0 i n}\right)$, à gauche pour $S_{i n}=0$, à droite pour $S_{\text {in }}=1$. 
Là aussi, la taille des régions de stabilité des équilibres augmente avec la concentration $S_{i n}$. Si $S_{i n}$ est élevée et $D$ est faible (région $J_{5}$ ) alors le système converge vers l'équilibre de coexistence $E^{*}$. Si le taux de dilution $D$ est important (région $J_{1}$ ), le système converge vers l'équilibre de lessivage $E_{l}$.

Nous définissons les fonctions :

$$
\begin{gathered}
G(D)=\frac{\left(\lambda_{S}-S_{i n}\right)\left(D+k_{h y d}\right)}{k_{0} k_{h y d}}, \quad L_{H}(D)=\frac{\lambda_{H}\left(D+k_{h y d}\right)}{\gamma_{s h} c_{s} k_{0} k_{h y d}}, \\
L_{A}(D)=\frac{\lambda_{A}\left(D+k_{h y d}\right)}{\gamma_{s a} c_{s} k_{0} k_{h y d}} \quad \text { et } \quad L_{V}(D)=\frac{\lambda_{V}\left(D+k_{h y d}\right)}{\gamma_{s v} c_{s} k_{0} k_{h y d}} .
\end{gathered}
$$

Si l'inégalité (3)est vérifiée, on peut montrer, en utilisant la proposition 2.3 , le résultat suivant :

Proposition 2.5. Le plan $\left(D, X_{0 i n}\right)$ est divisé en cinq régions décrites dans le tableau 4 :

\begin{tabular}{|c||c||c|c|c|c|c|c|c|c|c|}
\hline Condition & Région & $E_{l}$ & $E_{0}$ & $E_{H}$ & $E_{A}$ & $E_{A H}$ & $E_{V}$ & $E_{V H}$ & $E_{V A}$ & $E_{*}$ \\
\hline \hline$X_{0 i n}<G(D)$ & $J_{1}$ & GAS & & & & & & & & \\
\hline $\begin{array}{c}X_{0 i n}>G(D), \\
\text { et } X_{0 i n}<L_{H}(D)+G(D)\end{array}$ & $J_{2}$ & $\mathrm{I}$ & GAS & & & & & & & \\
\hline $\begin{array}{c}X_{0 i n}>L_{H}(D)+G(D), \\
\text { et } X_{0 i n}<L_{A}(D)+G(D)\end{array}$ & $J_{3}$ & $\mathrm{I}$ & $\mathrm{I}$ & $\mathrm{GAS}$ & & & & & & \\
\hline $\begin{array}{c}X_{0 i n}>L_{A}(D)+G(D), \\
\text { et } X_{0 i n}<L_{V}(D)+G(D)\end{array}$ & $J_{4}$ & $\mathrm{I}$ & $\mathrm{I}$ & $\mathrm{I}$ & $\mathrm{I}$ & $\mathrm{GAS}$ & & & & \\
\hline$X_{0 i n}>L_{V}(D)+G(D)$ & $J_{5}$ & $\mathrm{I}$ & $\mathrm{I}$ & $\mathrm{I}$ & $\mathrm{I}$ & $\mathrm{I}$ & $\mathrm{I}$ & $\mathrm{I}$ & $\mathrm{I}$ & GAS \\
\hline
\end{tabular}

Tableau 4. Existence et stabilité des équilibres du système (1)sans inhibition, dans le cas où $r_{0}=k_{h y d} X_{0}$, selon $\left(D, X_{0 i n}\right)$.

\subsubsection{Taux de biogaz produit}

A partir de l'analyse des équilibres du modèle, nous déterminons le taux du méthane et de l'hydrogène produits en chaque équilibre et nous identifions la voie qui donne une production maximale.

\subsubsection{Taux de méthane produit}

Le taux de méthane produit, en un équilibre $E$, est donné par la formule suivante :

$$
Q_{C H_{4}}=\alpha_{1} g_{A}\left(A_{\left.\right|_{A=A^{*}}}\right) X_{\left.A\right|_{X_{A}=X_{A}^{*}}}+\alpha_{2} g_{H}\left(H_{\left.\right|_{H=H^{*}}}\right) X_{\left.H\right|_{X_{H}=X_{H}^{*}}}
$$

avec $\alpha_{1}=\frac{1-c_{a}}{c_{a}}$ et $\alpha_{2}=\frac{1-c_{h}}{c_{h}}$.

Remarquons que d'après le schéma réactionnel de la Figure 1, le méthane $\mathrm{CH}_{4}$ est produit par les bactéries acétogènes $X_{A}$ ou par les bactéries méthanogènes hydrogénotrophes $X_{H}$. Pour avoir un maximum de méthane produit, il faut choisir $X_{A} \neq 0$ et $X_{H} \neq 0$. Les équilibres où les concentrations des biomasses $X_{A}$ et $X_{H}$ sont non nulles sont $E_{*}$ et $E_{A H}$. Donc, le taux de méthane produit peut être maximal soit à l'équilibre $E_{A H}$ ou à l'équilibre positif $E_{*}$.

On peut vérifier ce résultat en calculant le taux de méthane produit, pour chaque équilibre, voir le tableau 5 . 


\begin{tabular}{|c|c|}
\hline Équilibre & $Q_{C H_{4}}$ \\
\hline \hline$E_{l}, E_{0}$ et $E_{V}$ & 0 \\
\hline$E_{H}$ & $\alpha_{2} c_{h} D\left(H^{(0)}-\lambda_{H}\right)$ \\
\hline$E_{A}$ & $\alpha_{1} c_{a} D\left(A^{(0)}-\lambda_{A}\right)$ \\
\hline$E_{A H}$ & $\alpha_{1} c_{a} D\left(A^{(0)}-\lambda_{A}\right)+\alpha_{2} c_{h} D\left(H^{(0)}-\lambda_{H}\right)$ \\
\hline$E_{V H}$ & $\alpha_{2} c_{h} D\left(\bar{H}-\lambda_{H}\right)$ \\
\hline$E_{V A}$ & $\alpha_{1} c_{a} D\left(\bar{A}-\lambda_{A}\right)$ \\
\hline$E_{*}$ & $\alpha_{1} c_{a} D\left(\bar{A}-\lambda_{A}\right)+\alpha_{2} c_{h} D\left(\bar{H}-\lambda_{H}\right)$ \\
\hline
\end{tabular}

Tableau 5. Taux de méthane produit en chaque équilibre du modèle (1) sans inhibition, dans le cas où $r_{0}=k_{\text {hydr }} X_{0}$.

En effet, rappelons que $\bar{A}=A^{(0)}+\gamma_{v a} c_{v}\left(V^{(0)}-\lambda_{V}\right)$ et $\bar{H}=H^{(0)}+\gamma_{v h} c_{v}\left(V^{(0)}-\right.$ $\left.\lambda_{V}\right)$. Rappelons aussi que $E_{H}$ existe si $H^{(0)}>\lambda_{H}$ et que $E_{A}$ existe si $A^{(0)}>\lambda_{A}$. Sous ces deux conditions, $E_{A H}$ existe et le taux de méthane $Q_{C H_{4}}$ à l'équilibre $E_{A H}$ est plus grand que celui à l'équilibre $E_{A}$ et à l'équilibre $E_{H}$. De même, $E_{V H}$ existe si $\bar{H}>\lambda_{H}$ et $V^{(0)}>\lambda_{V}$ et $E_{V A}$ existe si $\bar{A}>\lambda_{A}$ et $V^{(0)}>\lambda_{V}$. On déduit que, si $E_{*}$ existe alors le taux de méthane $Q_{C H_{4}}$ à l'équilibre $E_{*}$ est plus grand que celui à l'équilibre $E_{V A}$ et à l'équilibre $E_{V H}$.En conclusion, on a :

- Si $V^{(0)}>\lambda_{V}$, alors $\bar{A}>A^{(0)}$ et $\bar{H}>H^{(0)}$. Dans ce cas, $E_{*}$ existe et est stable et le taux de méthane produit est maximal à l'équilibre $E_{*}$.

- Si $V^{(0)}<\lambda_{V}$ alors $\bar{A}<A^{(0)}$ et $\bar{H}<H^{(0)}$. Dans ce cas, $E_{A H}$ existe et est stable et le taux de méthane produit est maximal à l'équilibre $E_{A H}$.

On représente, dans la Figure 4, le taux maximal du méthane produit en fonction de la dilution $D$. On fait varier la valeur de la concentration du substrat sous forme particulaire $X_{0 i n}$ et a valeur de la concentration du substrat sous forme soluble $S_{i n}$ de telle manière à maintenir la somme $X_{0 i n}+S_{i n}$ constante.

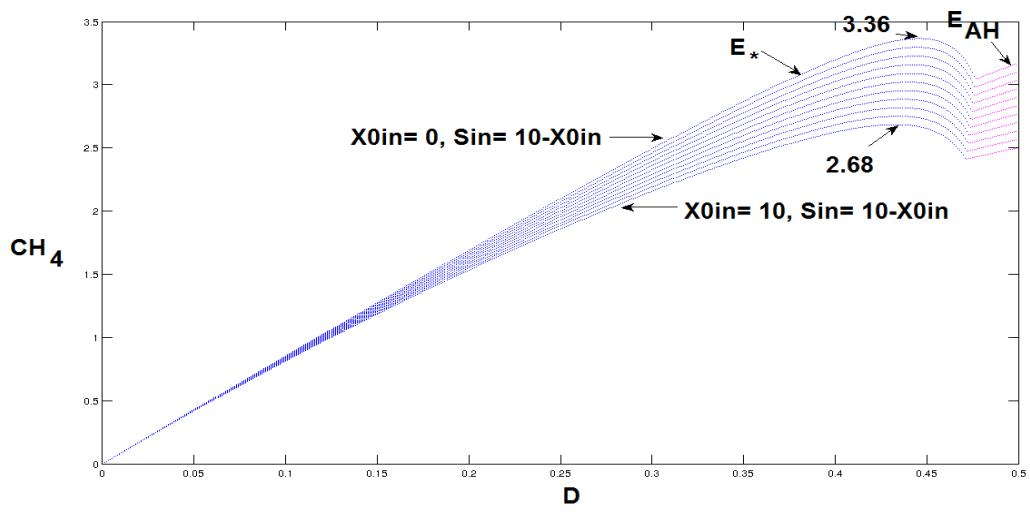

Figure 4. La variation des taux de méthane pour $S_{i n}+X_{0 i n}=10:$ Les courbes bleues représentent les taux de méthane à l'équilibre $E *$ et les courbes roses les taux de méthane à l'équilibre $E_{A H}$, selon les valeurs de $X_{0 i n}$ et de $S_{i n}$.

Dans la mesure où il existe un rendement de conversion du substrat solide en substrat soluble, selon que la matière organique se trouve sous l'une ou l'autre forme, la produc- 
tion de méthane diffère, voir Figure 4. Pour une même valeur de $X_{0 i n}+S_{i n}$, on produit plus de méthane en augmentant la concentration $S_{i n}$ du substrat sous forme soluble qu'en augmentant la concentration $X_{0 i n}$ du substrat sous forme particulaire.

Remarque : Si les valeurs des concentrations à l'entrée $S_{i n}$ et $X_{0 i n}$ sont plus élevées $\left(X_{0 i n}+S_{\text {in }}\right.$ est supérieure à la valeur 10), alors la courbe du taux maximal du méthane produit, en fonction de $D$, garde la même concavité et le maximum s'approche de la droite d'équation $D=\min \left(m_{s}, m_{v}, m_{a}, m_{h}\right)$. Mais, si ces concentrations sont assez faibles alors le maximum est plus proche de l'axe des ordonnées $D=0$.

\subsubsection{Taux d'hydrogène produit}

Le taux d'hydrogène produit est donné par la formule suivante :

$$
Q_{H 2}=\alpha_{3} g_{S}\left(S_{\left.\right|_{S=S^{*}}}\right) X_{S_{X_{S}=X_{S}^{*}}}+\alpha_{4} g_{V}\left(V_{\left.\right|_{V=V^{*}}}\right) X_{\left.V\right|_{X_{V}=X_{V}^{*}}}
$$

avec $\alpha_{3}=\frac{1-c_{s}}{c_{s}}$ et $\alpha_{4}=\frac{1-c_{v}}{c_{v}}$.

Le tableau 6 donne le taux d'hydrogène produit en chaque équilibre.

Nous pouvons donc déduire que :

\begin{tabular}{|c||c|}
\hline Équilibre & $Q_{H_{2}}$ \\
\hline \hline$E_{l}$ & 0 \\
\hline$E_{0}, E_{H}, E_{A}$ et $E_{A H}$ & $\alpha_{3} D X_{S}^{*}$ \\
\hline$E_{V}, E_{V H}, E_{V A}$ et $E_{*}$ & $\alpha_{3} D X_{S}^{*}+\alpha_{4} D c_{v}\left(V^{(0)}-\lambda_{V}\right)$ \\
\hline
\end{tabular}

Tableau 6. Taux d'hydrogène produit en chaque équilibre du modèle (1) sans inhibition, dans le cas où $r_{0}=k_{h y d r} X_{0}$.

1) Si $V^{(0)}>\lambda_{V}$ alors le taux d'hydrogène produit par le modèle sans inhibition est maximal à l'un des équilibres $E_{V}, E_{V H}, E_{V A}$ ou $E_{*}$, dès qu'il existe et est stable.

- Si $\bar{A}<\lambda_{A}$ et $\bar{H}<\lambda_{H}$ alors le taux d'hydrogène est maximal en $E_{V}$.

- Si $\bar{H}>\lambda_{H}$ et $\bar{A}<\lambda_{A}$ alors le taux d'hydrogène est maximal en $E_{V H}$.

- Si $\bar{A}>\lambda_{A}$ et $\bar{H}<\lambda_{H}$ alors le taux d'hydrogène est maximal en $E_{V A}$.

- Si $\bar{A}>\lambda_{A}$ et $\bar{H}>\lambda_{H}$ alors le taux d'hydrogène est maximal en $E_{*}$.

2) $\mathrm{Si} V^{(0)}<\lambda_{V}$ alors le taux maximal d'hydrogène produit est donné par $E_{0}, E_{H}$, $E_{A}$ ou $E_{A H}$ :

- Si $A^{(0)}<\lambda_{A}, H^{(0)}<\lambda_{H}$ et $S_{i n}^{*}>\lambda_{S}$ alors le taux d'hydrogène est maximal en $E_{0}$.

- Si $A^{(0)}<\lambda_{A}$ et $H^{(0)}>\lambda_{H}$ alors le taux d'hydrogène est maximal en $E_{H}$.

- Si $H^{(0)}<\lambda_{H}$ et $A^{(0)}>\lambda_{A}$ alors le taux d'hydrogène est maximal en $E_{A}$.

- Si $A^{(0)}>\lambda_{A}$ et $H^{(0)}>\lambda_{H}$ alors le taux d'hydrogène est maximal en $E_{A H}$.

Le taux maximal d'hydrogène produit est représenté, en fonction de $D$, dans la figure 5. Ici aussi, la production de l'hydrogène diffère, selon que la matière organique se trouve sous forme soluble ou particulaire, voir Figure 5. En maintenant la somme $X_{0 i n}+S_{i n}$ constante, le taux d'hydrogène est d'autant plus élevé que la concentration $S_{i n}$ est plus grande. 


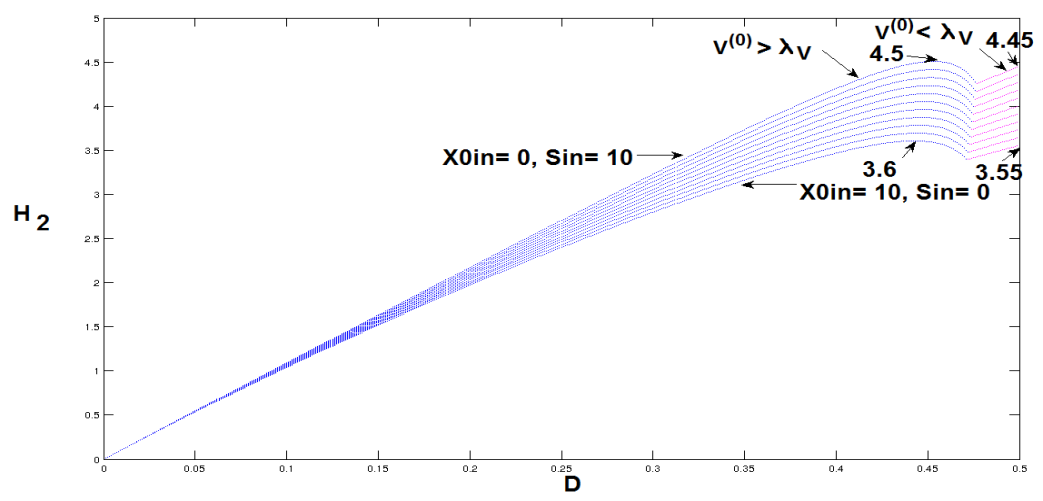

Figure 5. La variation du taux d'hydrogène pour $S_{\text {in }}+X_{0 i n}=10:$ Les courbes bleues représentent les taux d'hydrogène dans le cas où $V^{(0)}>\lambda_{V}$ et les courbes roses les taux d'hydrogène dans le cas où $V^{(0)}<\lambda_{V}$, selon les valeurs de $X_{0 i n}$ et de $S_{i n}$.

\subsection{Le modèle avec inhibition}

Dans ce modèle, on tient compte de l'inhibition de la croissance des bactéries acétogènes par un excès d'hydrogène dans le système et de celle des bactéries méthanogènes hydrogénotrophes et acétoclastes par l'acétate. On suppose alors que :

(H5) Pour tout $V \geqslant 0$ et $H \geqslant 0, g_{V}(0, H)=0, g_{V}(V, 0)>0, \frac{\partial g_{V}}{\partial V}(V, H)>0$, $\frac{\partial g_{V}}{\partial H}(V, H)<0$ et $\lim _{H \rightarrow+\infty} g_{V}(V, H)=0$.

(H6) Pour tout $H \geqslant 0$ et $A \geqslant 0, g_{H}(0, A)=0, g_{H}(H, 0)>0, \frac{\partial g_{H}}{\partial H}(H, A)>0$, $\frac{\partial g_{H}}{\partial A}(H, A)<0$ et $\lim _{H \rightarrow+\infty} g_{H}(H, A)=0$.

(H7) $g_{A}(0)=0$, il existe $A_{\max }>0$ tel que $g_{A}^{\prime}(A)>0$, pour $0<A<A_{\max }$ et $g_{A}^{\prime}(A)<0$, pour $A>A_{\text {max }}$ et $\lim _{A \longrightarrow+\infty} g_{A}(A)=0$.

Soient $\lambda_{V}(H)$ et $\lambda_{H}(A)$ les solutions respectives des équations $g_{V}\left(\lambda_{V}(H), H\right)=D$ et $g_{H}\left(\lambda_{H}(A), A\right)=D$. On note aussi par $\lambda_{A}^{i}, i=1,2$ les solutions de l'équation $g_{A}(A)=$ $D$, quand elles existent, avec $\lambda_{A}^{1}<\lambda_{A}^{2}$.

\subsubsection{Existence et stabilité des équilibres}

Comme dans la section 2.1, les trois premières équations du modèle (1) peuvent être découplées du reste du système. A l'équilibre, si $X_{S}=0$ alors $V=A=H=0$. Par suite, $X_{V}=X_{A}=X_{H}=0, S=S_{i n}^{*}:=\left(\frac{k_{0} k_{h y d}}{D+k_{h y d}}\right) X_{0 i n}+S_{i n}$ et $X_{0}=X_{0}^{*}:=$ $\left(\frac{D}{D+k_{h y d}}\right) X_{0 i n}$.

Mais, si $X_{S}>0$ alors $X_{0}=X_{0}^{*}, S=S^{*}:=\lambda_{S}$ et $X_{S}=X_{S}^{*}:=c_{s}\left(S_{i n}^{*}-\lambda_{S}\right)$. En 
remplaçant les variables $X_{0}, S$ et $X_{S}$ par leurs valeurs à l'équilibre dans les six dernières équations du système (1), on se ramène à l'étude du système (4) :

$$
\left\{\begin{aligned}
\frac{d V}{d t} & =D\left(V^{(0)}-V\right)-\frac{1}{c_{v}} g_{V}(V, H) X_{V} \\
\frac{d X_{V}}{d t} & =\left(g_{V}(V, H)-D\right) X_{V} \\
\frac{d A}{d t} & =D\left(A^{(0)}-A\right)+\gamma_{v a} g_{V}(V, H) X_{V}-\frac{1}{c_{a}} g_{A}(A) X_{A} \\
\frac{d X_{A}}{d t} & =\left(g_{A}(A)-D\right) X_{A} \\
\frac{d H}{d t} & =D\left(H^{(0)}-H\right)+\gamma_{v h} g_{V}(V, H) X_{V}-\frac{1}{c_{h}} g_{H}(H, A) X_{H} \\
\frac{d X_{H}}{d t} & =\left(g_{H}(H, A)-D\right) X_{H} .
\end{aligned}\right.
$$

où $V^{(0)}=\gamma_{s v} X_{S}^{*}, A^{(0)}=\gamma_{s a} X_{S}^{*}$ et $H^{(0)}=\gamma_{s h} X_{S}^{*}$.

Le sous-modèle (4) n'est autre que le système (9) de [11]. Il admet donc douze points d'équilibre dont deux équilibres strictement positifs, notés $E_{11}$ et $E_{12}$, voir le tableau 7, (où on a noté $E_{1}=\varepsilon_{0}, E_{2}=\varepsilon_{H}, E_{3}=\varepsilon_{A}^{1}, E_{4}=\varepsilon_{A}^{2}, E_{5}=\varepsilon_{A H}^{1}, E_{6}=\varepsilon_{A H}^{2}$, $E_{7}=\varepsilon_{V}, E_{8}=\varepsilon_{V H}, E_{9}=\varepsilon_{V A}^{1}, E_{10}=\varepsilon_{V A}^{2}, E_{11}=\varepsilon_{*}^{1}$ et $\left.E_{12}=\varepsilon_{*}^{2}\right)$

\begin{tabular}{|c|c|c|c|c|c|c|}
\hline Équilibre & $V$ & $X_{V}$ & $A$ & $X_{A}$ & $H$ & $X_{H}$ \\
\hline$E_{1}$ & $V^{(0)}$ & 0 & $A^{(0)}$ & 0 & $\bar{l}^{(0)}$ & 0 \\
\hline$E_{2}$ & $V^{(0)}$ & 0 & $A^{(0)}$ & 0 & $\lambda_{H}^{0}$ & $c_{h}\left(H^{(0)}-\lambda_{H}^{0}\right)$ \\
\hline$E_{3}$ & $V^{(0)}$ & 0 & $\lambda_{A}^{1}$ & $c_{a}\left(A^{(0)}-\lambda_{A}^{1}\right)$ & $H^{(0)}$ & 0 \\
\hline$E_{4}$ & $V^{(0)}$ & 0 & $\lambda_{A}^{2}$ & $c_{a}\left(A^{(0)}-\lambda_{A}^{2}\right)$ & $H^{(0)}$ & 0 \\
\hline$E_{5}$ & $V^{(0)}$ & 0 & $\lambda_{A}^{1}$ & $c_{a}\left(A^{(0)}-\lambda_{A}^{1}\right)$ & $\lambda_{H}^{1}$ & $c_{h}\left(H^{(0)}-\lambda_{H}^{1}\right)$ \\
\hline$E_{6}$ & $V^{(0)}$ & 0 & $\lambda_{A}^{2}$ & $c_{a}\left(A^{(0)}-\lambda_{A}^{2}\right)$ & $\lambda_{H}^{2}$ & $c_{h}\left(H^{(0)}-\lambda_{H}^{2}\right)$ \\
\hline$E_{7}$ & $\hat{\hat{V}}$ & $c_{v}\left(V^{(0)}-\hat{V}\right)$ & $\begin{array}{c}A_{V}^{(0)} \\
-\gamma_{v a} \hat{V}\end{array}$ & 0 & $\begin{array}{c}H_{V}^{(0)} \\
-\gamma_{v h} c_{v} \hat{V}\end{array}$ & 0 \\
\hline$E_{8}$ & $\breve{V}$ & $c_{v}\left(V^{(0)}-\breve{V}\right)$ & $\begin{array}{c}A_{V}^{(0)} \\
-\gamma_{v a} c_{v} \breve{V}\end{array}$ & 0 & $\breve{H}$ & $\begin{array}{c}c_{h}\left(H_{V}^{(0)}-\breve{H}\right. \\
\left.-\gamma_{v h} c_{v} \breve{V}\right)\end{array}$ \\
\hline$E_{9}$ & $\hat{V}$ & $c_{v}\left(V^{(0)}-\hat{V}\right)$ & $\lambda_{A}^{1}$ & $\begin{array}{c}c_{a}\left(A_{V}^{(0)}-\lambda_{A}^{1}\right. \\
\left.-\gamma_{v a} c_{v} \hat{V}\right)\end{array}$ & $\begin{array}{c}H_{V}^{(0)} \\
-\gamma_{v h} c_{v} \hat{V}\end{array}$ & 0 \\
\hline$E_{10}$ & $\hat{V}$ & $c_{v}\left(V^{(0)}-\hat{V}\right)$ & $\lambda_{A}^{2}$ & $\begin{array}{c}c_{a}\left(A_{V}^{(0)}-\lambda_{A}^{2}\right. \\
\left.-\gamma_{v a} c_{v} \hat{V}\right)\end{array}$ & $\begin{array}{c}H_{V}^{(0)} \\
-\gamma_{v h} c_{v} \hat{V}\end{array}$ & 0 \\
\hline$E_{11}$ & $\lambda_{V}^{1}$ & $c_{v}\left(V^{(0)}-\lambda_{V}^{1}\right)$ & $\lambda_{A}^{1}$ & $c_{a}\left(A^{1}-\lambda_{A}^{1}\right)$ & $\lambda_{H}^{1}$ & $c_{h}\left(H^{1}-\lambda_{H}^{1}\right)$ \\
\hline$E_{12}$ & $\lambda_{V}^{2}$ & $c_{v}\left(V^{(0)}-\lambda_{V}^{2}\right)$ & $\lambda_{A}^{2}$ & $c_{a}\left(A^{2}-\lambda_{A}^{2}\right)$ & $\lambda_{H}^{2}$ & $c_{h}\left(H^{2}-\lambda_{H}^{2}\right)$ \\
\hline
\end{tabular}

Tableau 7. Les équilibres du sous-modèle (4).

avec

- $\lambda_{V}^{0}=\lambda_{V}\left(H^{(0)}\right), \lambda_{H}^{0}=\lambda_{H}\left(A^{(0)}\right)$,

- $\lambda_{H}^{i}=\lambda_{H}\left(\lambda_{A}^{i}\right), \lambda_{V}^{i}=\lambda_{V}\left(\lambda_{H}^{i}\right)$ pour $i=1,2$,

- $A_{V}^{(0)}=A^{(0)}+\gamma_{v a} c_{v} V^{(0)}, H_{V}^{(0)}=H^{(0)}+\gamma_{v h} c_{v} V^{(0)}$,

- $A^{i}=A_{V}^{(0)}-\gamma_{v a} c_{v} \lambda_{V}^{i}, H^{i}=H_{V}^{(0)}-\gamma_{v h} c_{v} \lambda_{V}^{i}$ pour $i=1,2$,

- $\hat{V}$ est solution de l'équation implicite $\hat{V}=\lambda_{V}\left(H^{(0)}+\gamma_{v h} c_{v}\left(V^{(0)}-\hat{V}\right)\right)$, 
- $\hat{H}=H_{V}^{(0)}-\gamma_{v h} c_{v} \hat{V}, \hat{A}=A_{V}^{(0)}-\gamma_{v a} c_{v} \hat{V}$

- $\breve{H}=\lambda_{H}\left(A^{(0)}+\gamma_{v a} c_{v}\left(V^{(0)}-\lambda_{V}(\breve{H})\right)\right), \breve{V}=\lambda_{V}(\breve{H})$ et $\breve{A}=A_{V}^{(0)}-\gamma_{v a} c_{v} \breve{V}$.

Les conditions d'existence ainsi que les conditions de stabilité locale sont données, respectivement, dans les tableaux 8 et 9, voir [11, section 4.1], pour plus de détails.

\begin{tabular}{|c|c|}
\hline L'équilibre & Conditions d'existence \\
\hline \hline$E_{1}$ & toujours \\
\hline$E_{2}$ & $H^{(0)}>\lambda_{H}^{0}$ \\
\hline$E_{3}$ & $A^{(0)}>\lambda_{A}^{1}$ \\
\hline$E_{4}$ & $A^{(0)}>\lambda_{A}^{2}$ \\
\hline$E_{5}$ & $A^{(0)}>\lambda_{A}^{1}$ et $H^{(0)}>\lambda_{H}^{1}$ \\
\hline$E_{6}$ & $A^{(0)}>\lambda_{A}^{2}$ et $H^{(0)}>\lambda_{H}^{2}$ \\
\hline$E_{7}$ & $V^{(0)}>\lambda_{V}^{0}$ \\
\hline$E_{8}$ & $V^{(0)}>\lambda_{V}\left(\lambda_{H}^{0}\right)$ et $\breve{H}<H_{V}^{(0)}-\gamma_{v h} c_{v} \breve{V}$ \\
\hline$E_{9}$ & $V^{(0)}>\lambda_{V}^{0}$ et $\hat{A}>\lambda_{A}^{1}$ \\
\hline$E_{10}$ & $V^{(0)}>\lambda_{V}^{0}$ et $\hat{A}>\lambda_{A}^{2}$ \\
\hline$E_{11}$ & $V^{(0)}>\lambda_{V}^{1}, A^{1}>\lambda_{A}^{1}$ et $H^{1}>\lambda_{H}^{1}$ \\
\hline$E_{12}$ & $V^{(0)}>\lambda_{V}^{2}, A^{2}>\lambda_{A}^{2}$ et $H^{2}>\lambda_{H}^{2}$ \\
\hline
\end{tabular}

Tableau 8. Conditions d'existence des équilibres du sous-modèle (4).

\begin{tabular}{|c|c|}
\hline L'équilibre & Conditions de stabilité locale \\
\hline \hline$E_{1}$ & $V^{(0)}<\lambda_{V}^{0},\left(A^{(0)}<\lambda_{A}^{1}\right.$ ou $\left.A^{(0)}>\lambda_{A}^{2}\right)$ et $H^{(0)}<\lambda_{H}^{0}$ \\
\hline$E_{2}$ & $V^{(0)}<\lambda_{V}\left(\lambda_{H}^{0}\right),\left(A^{(0)}<\lambda_{A}^{1}\right.$ ou $\left.A^{(0)}>\lambda_{A}^{2}\right)$ \\
\hline$E_{3}$ & $V^{(0)}<\lambda_{V}^{0}$ et $H^{(0)}<\lambda_{H}^{1}$ \\
\hline$E_{4}$ & toujours instable \\
\hline$E_{5}$ & $V^{(0)}<\lambda_{V}^{1}$ \\
\hline$E_{6}$ & toujours instable \\
\hline$E_{7}$ & $\left(\hat{A}<\lambda_{A}^{1}\right.$ ou $\left.\hat{A}>\lambda_{A}^{2}\right)$ et $\hat{H}<\lambda_{H}(\hat{A})$ \\
\hline$E_{8}$ & $\vec{A}<\lambda_{A}^{1}$ ou $\breve{A}>\lambda_{A}^{2}$ \\
\hline$E_{9}$ & $\hat{H}<\lambda_{H}^{1}$ \\
\hline$E_{10}$ & toujours instable \\
\hline$E_{11}$ & stable lorsqu'il existe \\
\hline$E_{12}$ & toujours instable \\
\hline
\end{tabular}

Tableau 9. Conditions de stabilité locale des équilibres du sous-modèle (4). 


\subsubsection{Diagrammes opératoires}

On suppose, dans cette section, que les fonctions de croissance avec inhibition $g_{V}(.,$. et $g_{H}(.,$.$) sont données par les fonctions de Beddington suivantes, [4], voir aussi [11] et$ [12]:

$$
g_{V}(V, H)=\frac{m_{v} V}{k_{v}+V+\mu_{h} H} \quad \text { et } \quad g_{H}(H, A)=\frac{m_{h} H}{k_{h}+H+\mu_{a} A},
$$

$m_{v}, m_{h}$ et $k_{v}, k_{h}$ sont, respectivement, les taux de croissance maximale et les constantes de demisaturation, $\mu_{h}$ et $\mu_{a}$ sont des facteurs d'inhibition. Ces fonctions de croissance, utilisées par les biologistes, vérifient les hypothèses $(\mathbf{H 5})$ et $(\mathbf{H 6})$. La fonction de croissance $g_{A}$ est choisie de type Haldane :

$$
g_{A}(A)=\frac{m_{a} A}{k_{a}+A+\frac{A^{2}}{k_{I}}},
$$

$k_{I}$ est le coefficient d'inhibition de la croissance des bactéries méthanogènes acétoclastes $X_{A}$ par l'acétate. Lorsque la valeur de $k_{I}$ est très grande, la fonction de croissance microbienne $g_{A}(A)$ de type Haldane se comporte comme une fonction de type Monod. Les valeurs des paramètres utilisés sont celles du tableau A1 de [11].

Les figures 6 et 7 présentent les diagrammes opératoires du système (1) avec inhibition. On ne peut déterminer, dans ce cas, l'expression analytique des courbes délimitant les régions de stabilité des équilibres puisque les seuils de rentabilité sont les zéros de fonctions implicites et ne peuvent être déterminés explicitement. Les diagrammes sont donc obtenus par simulation du système pour plusieurs conditions initiales et les régions de stabilité sont dessinées point par point.

Pour une concentration $X_{0 i n}=0$, les diagrammes opératoires qu'on trouve sont ceux de [11], voir Figures 5-8.

Dans la figure 6 , on prend $X_{0 i n}=10$ et on suppose que les facteurs d'inhibition $\mu_{a}$ et $\mu_{h}$ sont nuls et que $k_{I}$ prend une très grande valeur $\left(k_{I}=100\right)$. On voit alors que les régions de stabilité pour de petites valeurs de $S_{i n}$ ont la même allure que celles trouvées pour le modèle sans inhibition. L'inhibition dans ce cas n'a pas d'effet sur le système. Par contre, pour des valeurs de $S_{\text {in }}$ entre 0 et 150 , une nouvelle région de stabilité apparaît, celle de l'équilibre de l'extinction des bactéries méthanogènes acétoclastes $E_{8}$. Il y a aussi apparition de régions de bistabilité : une première région où l'équilibre de $E_{8}$ et l'équilibre de coexistence $E_{11}$ sont stables (la région en bleu-ciel) et une seconde région où l'équilibre $E_{5}$ (extinction des bactéries acétogènes) et $E_{2}$ (extinction des bactéries méthanogènes acétoclastes et des bactéries acétogènes ) sont stables, (la région en mauve), voir Figure 6.

Dans la figure 7, le facteur $k_{I}$ est petit et les facteurs d'inhibition sont non nuls $\left(\mu_{a}=\mu_{h}=1\right)$. Il y a apparition d'une autre région de bistabilité, où $E_{11}$ et $E_{2}$ sont stables (la région en gris).

En augmentant, la valeur de la matière organique sous forme particulaire, $X_{0 i n}$, de 1 à 10 la région de stabilité de l'équilibre $E_{5}$ (la région en rose) et la région de bistabilité de $E_{5}$ et de $E_{2}$ (la région en mauve) disparaissent. Par contre, la région de stabilité de l'équilibre de coexistence $E_{11}$ (la région en noir) et la région de bistabilité de $E_{11}$ et de $E_{2}$ (la région en gris) augmentent de taille, voir Figure 7 à droite.

En augmentant la valeur de la concentration $X_{0 i n}$, on remarque que les régions de stabilité, dans le plan $\left(D, S_{i n}\right)$, translatent vers le bas et que la taille de certaines régions augmentent tandis que d'autres disparaissent. Chaque région correspond soit à un seul équilibre stable soit à un cas de bistabilité. 

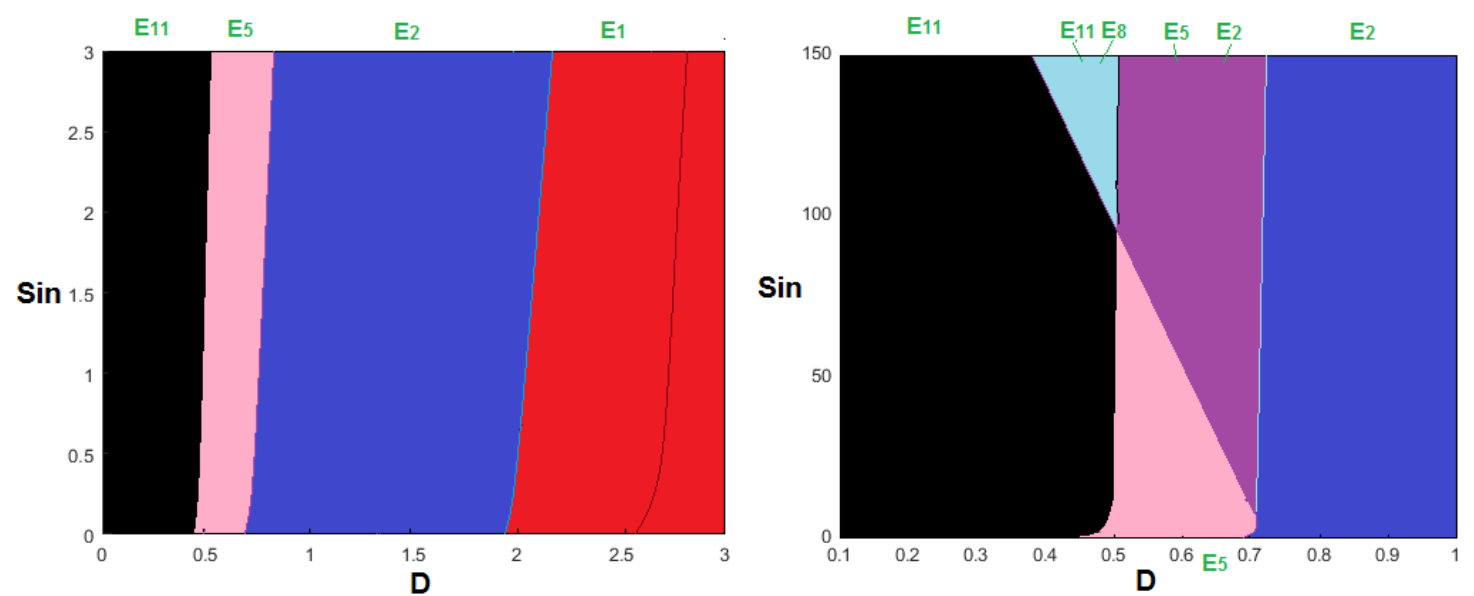

Figure 6. Diagramme opératoire du système (1) pour $X_{0 i n}=10$, avec $\mu_{a}=\mu_{h}=0$ et $k_{I}=100$, à droite pour $0 \leq S_{\text {in }} \leq 3$ et à gauche pour de grandes valeurs de $S_{i n}$.
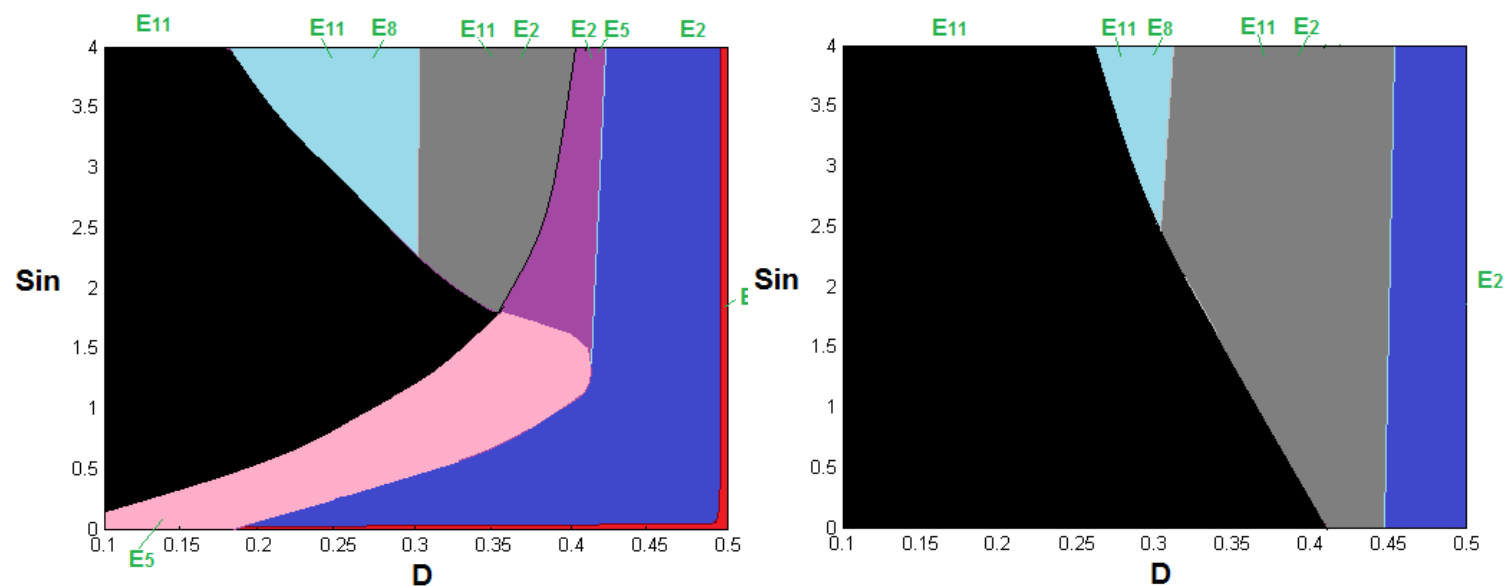

Figure 7. Diagramme opératoire du système (1) pour $X_{0 \text { in }}=1$ à gauche et $X_{0 \text { in }}=10$ à droite, avec $\mu_{a}=\mu_{h}=k_{I}=1$

Cette étude montre que l'inhibition a un impact sur la stabilité des équilibres et qu'en ajoutant l'étape d'hydrolyse, les régions de stabilité changent de taille et il y a apparition de nouvelles régions de stabilité et de bistabilité.

\subsubsection{Taux de biogaz produit pour le modèle avec inhibition}

Le taux de méthane (respectivement, de l'hydrogène) dépend des fonctions de croissance $g_{A}$ et $g_{H}$ (respectivement, de $g_{S}$ et de $g_{V}$ ). Ils diffèrent donc du cas sans inhibition.

\subsubsection{Taux de méthane produit}

Le taux de méthane produit en un équilibre $E$ est donné par la formule suivante :

$$
Q_{C H_{4}}(E)=\alpha_{1} g_{A}\left(A_{\left.\right|_{A=A^{*}}}\right) X_{\left.A\right|_{X_{A}=X_{A}^{*}}}+\alpha_{2} g_{H}\left(H_{\left.\right|_{H=H^{*}}}, A_{\left.\right|_{A=A^{*}}}\right) X_{\left.H\right|_{X_{H}=X_{H}^{*}}}
$$


Le tableau 10 donne le taux de méthane produit, pour chaque équilibre du modèle (1).

\begin{tabular}{|c|c|}
\hline Équilibre & $Q_{\mathrm{CH}_{4}}$ \\
\hline$E_{1}$ et $E_{7}$ & 0 \\
\hline$E_{2}$ & $\alpha_{2} c_{h} D\left(H^{(0)}-\lambda_{H}^{0}\right)$ \\
\hline$E_{3}$ & $\alpha_{1} c_{a} D\left(A^{(0)}-\lambda_{A}^{1}\right)$ \\
\hline$E_{4}$ & $\alpha_{1} c_{a} D\left(A^{(0)}-\lambda_{A}^{2}\right)$ \\
\hline$E_{5}$ & $\alpha_{1} c_{a} D\left(A^{(0)}-\lambda_{A}^{1}\right)+\alpha_{2} c_{h} D\left(H^{(0)}-\lambda_{H}^{1}\right)$ \\
\hline$E_{6}$ & $\alpha_{1} c_{a} D\left(A^{(0)}-\lambda_{A}^{2}\right)+\alpha_{2} c_{h} D\left(H^{(0)}-\lambda_{H}^{2}\right)$ \\
\hline$E_{8}$ & $\alpha_{2} D c_{h}\left(H_{V}^{(0)}-\breve{H}-\gamma_{v h} c_{v} \breve{V}\right)$ \\
\hline$E_{9}$ & $\alpha_{1} D c_{a}\left(A_{V}^{(0)}-\lambda_{A}^{1}-\gamma_{v a} c_{v} \hat{V}\right)$ \\
\hline$E_{10}$ & $\alpha_{1} D c_{a}\left(A_{V}^{(0)}-\lambda_{A}^{2}-\gamma_{v a} c_{v} \hat{V}\right)$ \\
\hline$E_{11}$ & $\alpha_{1} D c_{a}\left(A^{1}-\lambda_{A}^{1}\right)+\alpha_{2} D c_{h}\left(H^{1}-\lambda_{H}^{1}\right)$ \\
\hline$E_{12}$ & $\alpha_{1} D c_{a}\left(A^{2}-\lambda_{A}^{2}\right)+\alpha_{2} D c_{h}\left(H^{2}-\lambda_{H}^{2}\right)$ \\
\hline
\end{tabular}

Tableau 10. Taux de méthane produit en chaque équilibre du modèle (1) avec hydrolyse enzymatique et inhibition, voir (5), (6) et (7).

Les seuils de rentabilité $\lambda_{A}^{1}, \lambda_{A}^{2}$ sont définis, pour $D<\frac{m_{a}}{1+2 \sqrt{\frac{k_{a}}{k_{I}}}}$ par :

$\lambda_{A}^{1}=\frac{\left(m_{a}-D\right)-\sqrt{\left(D-m_{a}\right)^{2}-4 D^{2} \frac{k_{a}}{k_{I}}}}{\frac{2 D}{k_{I}}}, \quad \lambda_{A}^{2}=\frac{\left(m_{a}-D\right)+\sqrt{\left(D-m_{a}\right)^{2}-4 D^{2} \frac{k_{a}}{k_{I}}}}{\frac{2 D}{k_{I}}}$.

$\lambda_{H}^{1}, \lambda_{H}^{2}, \lambda_{V}^{1}$ et $\lambda_{V}^{2}$ sont définis par :

$$
\begin{gathered}
\lambda_{H}^{1}=\frac{D\left(k_{h}+\mu_{a} \lambda_{A}^{1}\right)}{m_{h}-D}, \quad \lambda_{H}^{2}=\frac{D\left(k_{h}+\mu_{a} \lambda_{A}^{2}\right)}{m_{h}-D}, \quad \text { pour } \quad D<\min \left(m_{h}, \frac{m_{a}}{1+2 \sqrt{\frac{k_{a}}{k_{I}}}}\right), \\
\lambda_{V}^{1}=\frac{D\left(k_{v}+\mu_{h} \lambda_{H}^{1}\right)}{m_{v}-D}, \quad \lambda_{V}^{2}=\frac{D\left(k_{v}+\mu_{h} \lambda_{H}^{2}\right)}{m_{v}-D}, \text { pour } D<\min \left(m_{v}, m_{h}, \frac{m_{a}}{1+2 \sqrt{\frac{k_{a}}{k_{I}}}}\right) .
\end{gathered}
$$

Notons que si $X_{A}=0$ alors l'acétate $A$ s'accumule dans le milieu. Par suite, la croissance des bactéries méthanogènes hydrogénotrophes $X_{H}$ sera aussi inhibée, ce qui inhibe la production du méthane. Maintenant, si $X_{H}=0$ alors l'hydrogène $H_{2}$ s'accumule dans le milieu, ce qui inhibe la croissance des bactéries acétogènes $X_{V}$. Par suite, la production de l'acétate $A$ et de l'hydrogène $H$ diminue, ainsi que celle du méthane.

Le taux maximal de méthane est donc atteint aux équilibres où les biomasses $X_{A} \neq 0$ et $X_{H} \neq 0$, c'est à dire aux équilibres $E_{5}, E_{6}, E_{11}$ et $E_{12}$. Comme $Q_{C H_{4}}\left(E_{5}\right)>Q_{C H_{4}}\left(E_{6}\right)$ et $Q_{C H_{4}}\left(E_{11}\right)>$ $Q_{C H_{4}}\left(E_{12}\right)$ alors le taux maximal du méthane produit peut être donné soit par l'équilibre $E_{5}$, soit par l'équilibre $E_{11}$.

D'après le tableau 10 , Si $A^{(0)}>\max \left(\lambda_{A}^{1}, A^{1}\right)$ et $H^{(0)}>\max \left(\lambda_{H}^{1}, H^{1}\right)$, le taux maximal du méthane produit est donné par l'équilibre $E_{5}$.

Dans le cas où $A^{1}>\max \left(\lambda_{A}^{1}, A^{(0)}\right)$ et $H^{1}>\max \left(\lambda_{H}^{1}, H^{(0)}\right)$, le taux maximal du méthane produit est donné par l'équilibre $E_{11}$.

Notons que si $A^{(0)}>\lambda_{A}^{1}$ et $H^{(0)}<\lambda_{H}^{1}$ alors le taux maximal du méthane produit est donné par l'équilibre $E_{3}$, puisque dans ce cas $E_{5}$ est instable et $E_{11}$ n'existe pas, voir Figures 8, 9 et 10. 
Pour les simulations suivantes, on prend $\mu_{h}=\mu_{a}=1, k_{I}=100, k_{0}=1$ et $k_{h y d}=2$. On représente dans les figures 8,9 et 10 le taux du méthane produit, pour $D<\min \left(m_{s}, m_{h}, \frac{m_{a}}{1+2 \sqrt{\frac{k_{a}}{k_{I}}}}\right)$. On fait varier $X_{0 i n}$ entre 0 et 10 tout en maintenant la somme $X_{0 i n}+S_{i n}=10$.

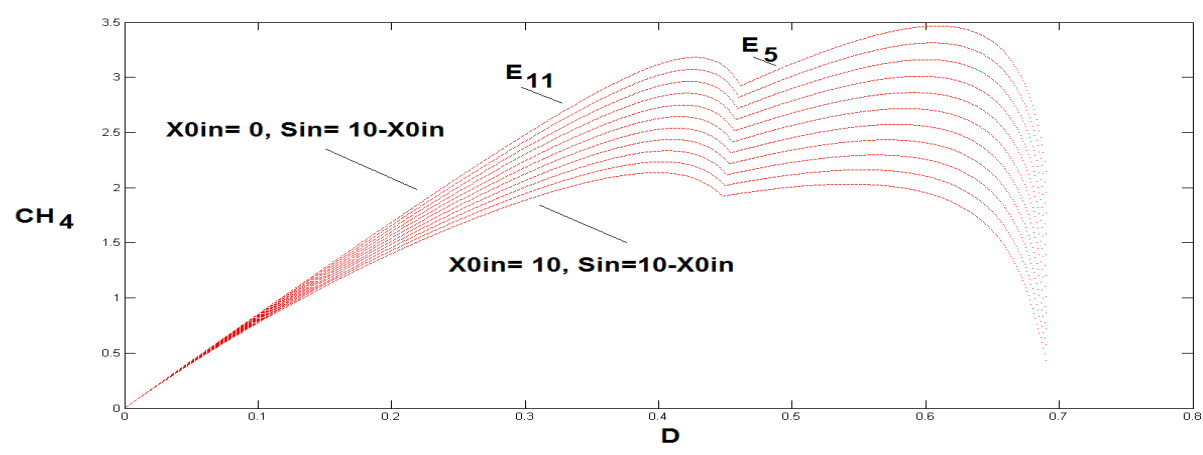

Figure 8. La variation du taux maximal de méthane pour $S_{\text {in }}+X_{0 \text { in }}=10,0 \leqslant D \leqslant 0.7$, $\mu_{h}=\mu_{a}=1$ et $k_{I}=100$

En prenant d'autres valeurs de paramètres, $k_{I}=10, \mu_{h}=1$ et $\mu_{a}=5$, on trace le taux de méthane produit pour $X_{0 i n}+S_{i n}=10$, voir Figure 9 .

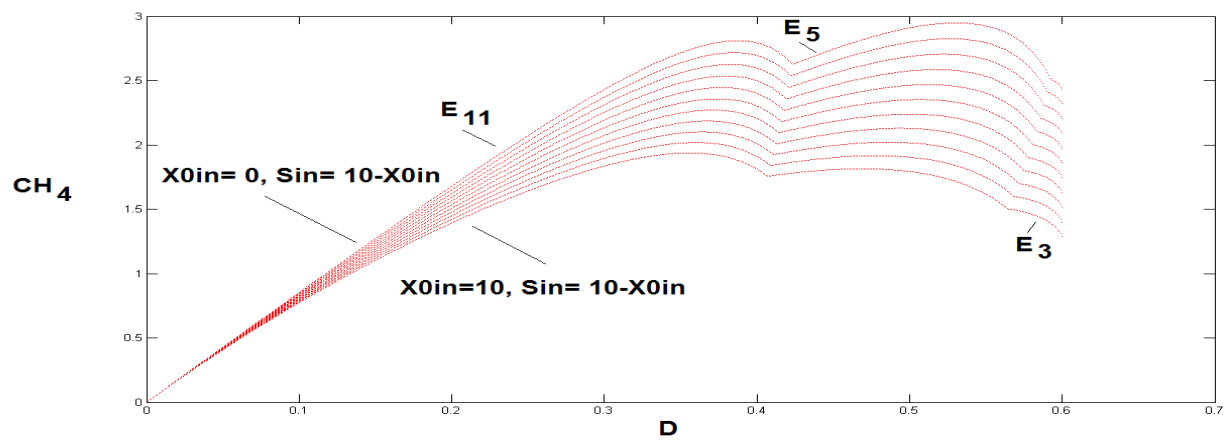

Figure 9. La variation du taux maximal du méthane pour $S_{\text {in }}+X_{0 \text { in }}=10,0 \leqslant D \leqslant 0.6$, $\mu_{h}=1, \mu_{a}=5$ et $k_{I}=10$.

Avec ce nouveau jeu de paramètres, le taux maximal de biogaz est toujours donné par l'un des équilibres $E_{11}$ ou $E_{5}$. Lorsque, les paramètres $\mu_{a}$ et/ou $\mu_{h}$ augmentent et $k_{I}$ diminue, l'inhibition augmente et on produit moins de méthane. En effet, dans la figure 9, on voit que le taux maximal de biogaz est inférieur à celui représenté dans la figure 8 .

On diminue à présent la concentration des substrats à l'entrée et on représente le taux de méthane produit pour $S_{i n}+X_{0 i n}=5$, sans changer les facteurs d'inhibition : $k_{I}=10, \mu_{h}=1$ et $\mu_{a}=5$.

Pour $X_{0 i n}=0$ et $S_{i n}=5$, voir Figure 10, le maximum global du taux de méthane est donné par l'équilibre $E_{5}$. Ce résultat est analogue à celui trouvé dans [12], voir Figure 5 (c). Dans cette figure, on note que le taux de biogaz produit est légèrement inférieur à celui illustré dans la figure 10, puisque nous ne tenons pas compte des termes de mortalité des biomasses. 


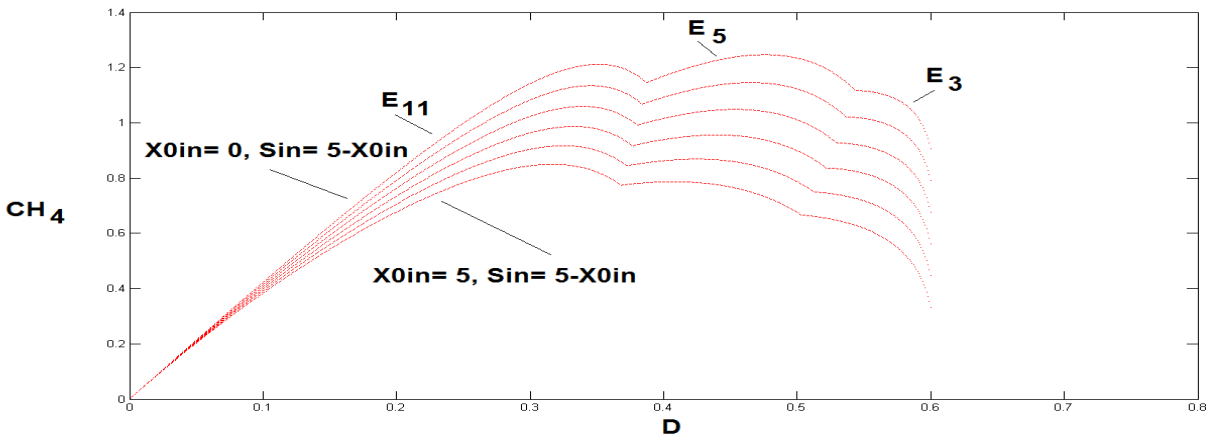

Figure 10. La variation du taux maximal du méthane pour $S_{i n}=5-X_{0 i n}, 0 \leqslant D \leqslant 0.6$, $\mu_{h}=1, \mu_{a}=5$ et $k_{I}=10$.

En tenant compte de l'étape d'hydrolyse et en augmentant le substrat à l'entrée $X_{0 i n}+S_{i n}$ de 5 à 10, voir Figure 10 et Figure 9, on remarque que le taux de biogaz produit est plus important dans la figure 9 que dans la figure 10, (il peut atteindre le double). Le taux de méthane produit dépend donc de l'étape d'hydrolyse qu'on ne peut négliger dans le processus de digestion anaérobie.

\subsubsection{Taux d'hydrogène produit}

Le taux d'hydrogène produit est donné par la formule suivante :

$$
Q_{H_{2}}=\alpha_{3} g_{S}\left(S_{\left.\right|_{S=S^{*}}}\right) X_{S_{X_{X}}=X_{S}^{*}}+\alpha_{4} g_{V}\left(V_{\left.\right|_{V=V^{*}}}, H_{\left.\right|_{H=H^{*}}}\right) X_{\left.V\right|_{X_{V}=X_{V}^{*}}}
$$

On calcule pour chaque équilibre, le taux d'hydrogène produit, voir tableau 11.

\begin{tabular}{|c||c|}
\hline Équilibre & $Q_{H_{2}}$ \\
\hline \hline$E_{1}, E_{2}, E_{3}, E_{4}, E_{5}$ et $E_{6}$ & $\alpha_{3} D X_{S}^{*}$ \\
\hline$E_{7}, E_{9}$ et $E_{10}$ & $\alpha_{3} D X_{S}^{*}+\alpha_{4} D c_{v}\left(V^{(0)}-\hat{V}\right)$ \\
\hline$E_{8}$ & $\alpha_{3} D X_{S}^{*}+\alpha_{4} D c_{v}\left(V^{(0)}-V\right)$ \\
\hline$E_{11}$ & $\alpha_{3} D X_{S}^{*}+\alpha_{4} D c_{v}\left(V^{(0)}-\lambda_{V}^{1}\right)$ \\
\hline$E_{12}$ & $\alpha_{3} D X_{S}^{*}+\alpha_{4} D c_{v}\left(V^{(0)}-\lambda_{V}^{2}\right)$ \\
\hline
\end{tabular}

Tableau 11. Taux d'hydrogène produit pour chaque équilibre du modèle (1) avec hydrolyse enzymatique et inhibition.

Comme les équilibres $E_{4}$ et $E_{6}$ sont instables dès qu'ils existent, le taux maximal d'hydrogène produit est donné, dans le cas où $V^{(0)}<\hat{V}, V^{(0)}<\hat{V}$ et $V^{(0)}<\lambda_{V}^{1}$, par l'un des équilibres $E_{1}$, $E_{2}, E_{3}$ ou $E_{5}$. Les équilibres $E_{7}, \cdots, E_{12}$ n'existent pas dans ce cas.

Dans le cas où $V^{(0)}>\hat{V}, V^{(0)}<\breve{V}$ et $V^{(0)}<\lambda_{V}^{1}$, l'équilibre $E_{10}$ est instable et le taux maximal d'hydrogène produit est donné par $E_{7}$ ou $E_{9}$.

Dans le cas $V^{(0)}<\hat{V}, V^{(0)}>\breve{V}$ et $V^{(0)}<\lambda_{V}^{1}$, le taux maximal d'hydrogène produit est donné par l'équilibre $E_{8}$.

On représente, dans la figure 11, le taux d'hydrogène $Q_{H_{2}}=\alpha_{3} D X_{S}^{*}$, pour $V^{(0)}<\hat{V}$, $V^{(0)}<\breve{V}$ et $V^{(0)}<\lambda_{V}^{1}$ et $X_{0 i n}+S_{i n}=10$. Dans ce cas, les taux d'hydrogène donnés par les équilibres $E_{1}, E_{2}, E_{3}$ ou $E_{5}$ sont égaux et correspondent au taux maximal. 


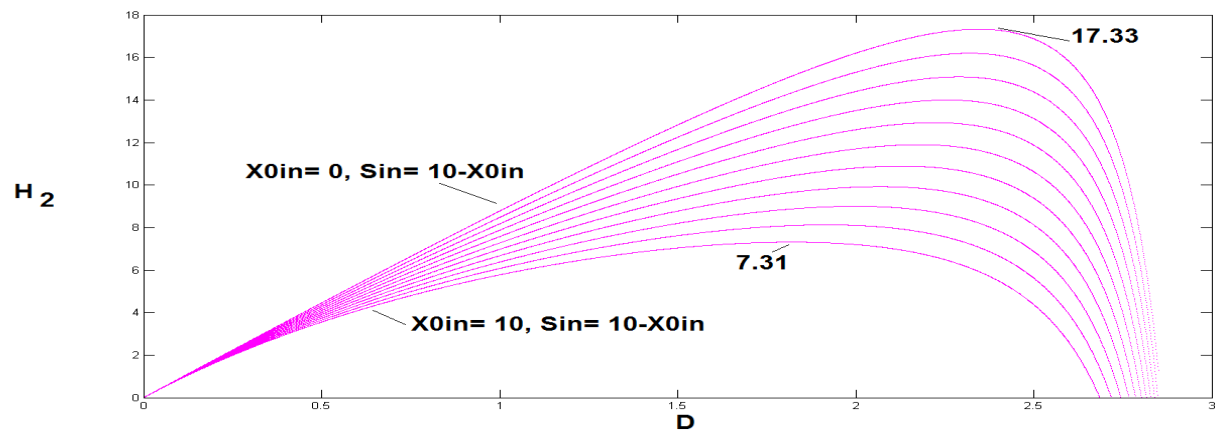

Figure 11. La variation du taux d'hydrogène pour $V^{(0)}<\hat{V}, V^{(0)}<\breve{V}$ et $V^{(0)}<\lambda_{V}^{1}$ et $S_{i n}+X_{0 i n}=10$.

\section{L'analyse d'un modèle avec compartiment microbien hydrolytique}

On considère, dans un second temps, le modèle (1) en supposant que $r_{0}=g_{0}\left(X_{0}\right) X_{S}$. Notons que les trois premières équations du modèle (1) peuvent se découpler des autres équations. On s'intéresse donc, dans un premier temps, au sous-modèle :

$$
\left\{\begin{aligned}
\frac{d X_{0}}{d t} & =D\left(X_{0 i n}-X_{0}\right)-g_{0}\left(X_{0}\right) X_{S} \\
\frac{d S}{d t} & =D\left(S_{i n}-S\right)-\frac{1}{c_{s}} g_{S}(S) X_{S}+k_{0} g_{0}\left(X_{0}\right) X_{S} \\
\frac{d X_{S}}{d t} & =\left(g_{S}(S)-D\right) X_{S}
\end{aligned}\right.
$$

On suppose que :

(H8) $\forall 0<X_{0}<X_{0 i n}, g_{0}(0)=0, g_{0}^{\prime}\left(X_{0}\right)>0$ et $g_{0}^{\prime \prime}\left(X_{0}\right) \leqslant 0$.

L'hypothèse (H8) est vérifiée pour les fonctions de croissance linéaires et celles de type Monod.

\subsection{L'analyse du sous-modèle (8)}

On peut vérifier facilement que les solutions du système (8) vérifient la propriété suivante :

Proposition 3.1. Pour des conditions initiales positives, les solutions du système (8) restent positives et bornées, pour tout $t \geq 0$.

Le sous-modèle (8) correspond au modèle (3) de [2] où $k_{1}=\frac{1}{c_{s}}$ et $\alpha=1$. Nous rappelons ici les principales étapes de l'étude. L'équilibre de lessivage où $X_{S}=0$ est donné par : $F_{0}=\left(X_{0 i n}, S_{i n}, 0\right)$. Cet équilibre existe toujours. Maintenant, si $X_{S} \neq 0$ alors $S=\lambda_{S}, X_{0}$ est l'intersection de la courbe de la fonction :

$$
\left.\zeta\left(X_{0}\right)=\frac{D\left(X_{0 i n}-X_{0}\right)}{g_{0}\left(X_{0}\right)}, \text { pour } X_{0} \in\right] 0, X_{0 i n}[
$$

et de la droite $\Delta$ d'équation : $\delta\left(X_{0}\right)=c_{s}\left[\left(S_{i n}-\lambda_{S}\right)+k_{0}\left(X_{0 i n}-X_{0}\right)\right]$ et $X_{S}=\delta\left(X_{0}\right)$. La recherche des équilibres strictement positifs revient à trouver les zéros de la fonction

$$
\left.H\left(X_{0}\right)=\zeta\left(X_{0}\right)-\delta\left(X_{0}\right), \text { pour } X_{0} \in\right] 0, X_{0 i n}[.
$$

Notons que $H^{\prime}\left(X_{0}\right)=0$ si et seulement si $\zeta^{\prime}\left(X_{0}\right)=-c_{s} k_{0}$. 
Lemme 3.1. Sous l'hypothèse (H8), $\zeta$ s'annule pour $X_{0}=X_{0 i n}$, et est décroissante et convexe.

Le lemme 3.1 implique le résultat suivant :

Lemme 3.2. L'équation, $\zeta^{\prime}\left(X_{0}\right)=-c_{s} k_{0}$ admet une unique solution $0<\bar{X}_{0}<X_{0 i n}$ si et seulement si $\zeta^{\prime}\left(X_{0 i n}\right)>-c_{s} k_{0}$.

On note que :

$$
\zeta^{\prime}\left(X_{0 i n}\right)>-c_{s} k_{0} \Leftrightarrow g_{0}\left(X_{0 i n}\right)>\frac{D}{c_{s} k_{0}} .
$$

Pour déterminer les équilibres strictement positifs du système (8), nous devons distinguer les deux cas : le cas où $g_{0}\left(X_{0 i n}\right) \leqslant \frac{D}{c_{s} k_{0}}$ et le cas où $g_{0}\left(X_{0 i n}\right)>\frac{D}{c_{s} k_{0}}$.

3.1.1. Le cas $g_{0}\left(X_{0 i n}\right) \leqslant \frac{D}{c_{s} k_{0}}$

Dans ce cas, l'intersection entre $\Delta$ et le graphe de $\zeta$ est soit vide, soit un unique point $\left(X_{0}^{*}, X_{S}^{*}\right)$ qui correspond à l'équilibre $F_{1}^{*}=\left(X_{0}^{*}, \lambda_{S}, X_{S}^{*}\right)$. En effet, $H$ est décroissante sur $] 0, X_{0 i n}[$, $H\left(X_{0 i n}\right)=-c_{s}\left(S_{i n}-\lambda_{S}\right)$ et $\lim _{X_{0} \rightarrow 0} H\left(X_{0}\right)=+\infty$. Nous pouvons donc déduire que :

Proposition 3.2. Sous les hypothèses $(\mathbf{H 1})$ et $(\mathbf{H 8})$, on a :

1) Si $S_{i n}>\lambda_{S}$ alors il existe un unique équilibre strictement positif $F_{1}^{*}=\left(X_{0}^{*}, \lambda_{S}, X_{S}^{*}\right)$ où $X_{0}^{*}$ est la solution de $\zeta\left(X_{0}\right)=\delta\left(X_{0}\right)$ et $X_{S}^{*}=\delta\left(X_{0}^{*}\right)$.

2) Si $S_{i n} \leqslant \lambda_{S}$ alors il n'existe pas d'équilibre strictement positif.

On peut remarquer que, lorsque $S_{i n}=\lambda_{S}$ alors $F_{1}^{*}$ coïncide avec $F_{0}$.

En calculant les valeurs propres de la matrice Jacobienne en $F_{0}$ et en utilisant le critère de Routh-Hurwitz pour l'équilibre $F_{1}^{*}$, on peut montrer le résultat suivant :

Proposition 3.3. Sous les hypothèses (H1) et (H8), les conditions d'existence et de stabilité des équilibres du système (8) sont données par:

\begin{tabular}{|c||c|c|}
\hline L'équilibre & Conditions d'existence & Conditions de stabilité locale \\
\hline \hline$F_{0}=\left(X_{0 i n}, S_{i n}, 0\right)$ & existe toujours & $S_{i n}<\lambda_{S}$ \\
\hline$F_{1}^{*}=\left(X_{0}^{*}, \lambda_{S}, X_{S}^{*}\right)$ & $S_{i n}>\lambda_{S}$ & lorsqu'il existe \\
\hline
\end{tabular}

Tableau 12. Les conditions d'existence et de stabilité des équilibres du système (8), dans le cas $g_{0}\left(X_{0 i n}\right) \leqslant \frac{D}{c_{s} k_{0}}$.

\subsubsection{Le cas $g_{0}\left(X_{0 i n}\right)>\frac{D}{c_{s} k_{0}}$}

Dans ce cas, si l'intersection de $\Delta$ et du graphe de $\zeta$ n'est pas vide alors elle comporte un ou deux points. L'équation $\zeta^{\prime}\left(X_{0}\right)=-c_{s} k_{0}$ représente le cas où $\Delta$ est tangente au graphe de $\zeta$. Cette équation admet une unique solution notée $\bar{X}_{0}$. Soit $\bar{X}_{S}=\zeta\left(\bar{X}_{0}\right)$ et $\bar{F}_{1}=\left(\bar{X}_{0}, \lambda_{S}, \bar{X}_{S}\right)$ l'équilibre strictement positif correspondant. On note par $\bar{S}_{i n}$ la valeur de $S_{i n}$ pour laquelle $\bar{X}_{S}=\zeta\left(\bar{X}_{0}\right)$. D'où, $\bar{S}_{i n}=\left(\frac{\bar{X}_{S}}{c_{s}}+k_{0} \bar{X}_{0}+\lambda_{S}\right)-k_{0} X_{0 i n}$. On peut donc montrer le résultat suivant :

Proposition 3.4. On suppose que

1) Si $\lambda_{S} \leqslant S_{i n}$ alors il existe un unique équilibre strictement positif $F_{1}^{*}$.

2) $S i \max \left(0, \bar{S}_{i n}\right)<S_{i n}<\lambda_{S}$ alors il existe deux équilibres strictement positifs $F_{1}^{*}$ et $F_{1}^{* *}$

3) Si $S_{i n}=\bar{S}_{i n}$ alors il existe un unique équilibre strictement positif $\bar{F}_{1}$.

4) Si $S_{i n}<\max \left(0, \bar{S}_{i n}\right)$ alors il n'existe pas d'équilibre strictement positif. 
En calculant les valeurs propres de la matrice Jacobienne en $F_{0}$ et en utilisant le critère de Routh-Hurwitz pour l'équilibre $F_{1}^{*}$ et $F_{1}^{* *}$, on peut montrer le résultat suivant :

Proposition 3.5. Sous les hypothèses (H1) et (H8), les conditions d'existence et de stabilité des équilibres du système (8) sont données par:

\begin{tabular}{|c||c|c|}
\hline L'équilibre & Conditions d'existence & Conditions de stabilité locale \\
\hline \hline$F_{0}=\left(X_{0 i n}, S_{i n}, 0\right)$ & existe toujours & $S_{i n}<\lambda_{S}$ \\
\hline$F_{1}^{*}=\left(X_{0}^{*}, \lambda_{S}, X_{S}^{*}\right)$ & $S_{i n}>\max \left(0, \bar{S}_{i n}\right)$ & $\zeta^{\prime}\left(X_{0}^{*}\right)<-c_{s} k_{0}$ \\
\hline$F_{1}^{* *}=\left(X_{0}^{* *}, \lambda_{S}, X_{S}^{* *}\right)$ & $\max \left(0, S_{i n}\right)<S_{i n}<\lambda_{S}$ & instable \\
\hline
\end{tabular}

Tableau 13. Les conditions d'existence et de stabilité des équilibres du système (8), dans le cas $g_{0}\left(X_{0 i n}\right)>\frac{D}{c_{s} k_{0}}$.

\subsection{L'analyse du modèle complet de la digestion anaérobie}

On suppose, dans cette section, que $g_{V}(V)=g_{V}(V, 0), g_{H}(H)=g_{H}(H, 0)$ et que $g_{A}$ vérifie l'hypothèse (H3).

\subsubsection{L'analyse des équilibres}

A partir de l'étude du sous modèle (8) et en utilisant la méthode utilisée dans la section 3.1, nous pouvons déduire les équilibres du modèle complet sans inhibition (1), avec $r_{0}=g_{0}\left(X_{0}\right) X_{S}$.

Proposition 3.6. Sous les hypothèses (H1) - (H4) et (H8), les équilibres du système (1) sont donnés dans le tableau 14

\begin{tabular}{|c|c|c|c|c|c|c|c|c|c|}
\hline Équilibre & $X_{0}$ & $S$ & $X_{S}$ & $V$ & $X_{V}$ & $A$ & $X_{A}$ & $H$ & $X_{H}$ \\
\hline$F_{l}$ & $X_{0 i n}$ & $S_{i n}$ & 0 & 0 & 0 & 0 & 0 & 0 & 0 \\
\hline$F_{0}^{*}$ & $X_{0}^{*}$ & $\lambda_{S}$ & $X_{S}^{*}$ & $V^{(0)}$ & 0 & $A^{(0)}$ & 0 & $H^{(0)}$ & 0 \\
\hline$F_{H}^{*}$ & $X_{0}^{*}$ & $\lambda_{S}$ & $X_{S}^{*}$ & $V^{(0)}$ & 0 & $A^{(0)}$ & 0 & $\lambda_{H}$ & $c_{h}\left(H^{(0)}-\lambda_{H}\right)$ \\
\hline$F_{A}^{*}$ & $X_{0}^{*}$ & $\lambda_{S}$ & $X_{S}^{*}$ & $V^{(0)}$ & 0 & $\lambda_{A}$ & $c_{a}\left(A^{(0)}-\lambda_{A}\right)$ & $H^{(0)}$ & 0 \\
\hline$F_{A H}^{*}$ & $X_{0}^{*}$ & $\lambda_{S}$ & $X_{S}^{*}$ & $V^{(0)}$ & 0 & $\lambda_{A}$ & $c_{a}\left(A^{(0)}-\lambda_{A}\right)$ & $\lambda_{H}$ & $c_{h}\left(H^{(0)}-\lambda_{H}\right)$ \\
\hline$F_{V}^{*}$ & $X_{0}^{*}$ & $\lambda_{S}$ & $X_{S}^{*}$ & $\lambda_{V}$ & $c_{v}\left(V^{(0)}-\lambda_{V}\right)$ & $\bar{A}$ & 0 & $\bar{H}$ & 0 \\
\hline$F_{V H}^{*}$ & $X_{0}^{*}$ & $\lambda_{S}$ & $X_{S}^{*}$ & $\lambda_{V}$ & $c_{v}\left(V^{(0)}-\lambda_{V}\right)$ & $\bar{A}$ & 0 & $\lambda_{H}$ & $c_{h}\left(\bar{H}-\lambda_{H}\right)$ \\
\hline$F_{V A}^{*}$ & $X_{0}^{*}$ & $\lambda_{S}$ & $X_{S}^{*}$ & $\lambda_{V}$ & $c_{v}\left(V^{(0)}-\lambda_{V}\right)$ & $\lambda_{A}$ & $c_{a}\left(\bar{A}-\lambda_{A}\right)$ & $\bar{H}$ & 0 \\
\hline$F^{*}$ & $X_{0}^{*}$ & $\lambda_{S}$ & $X_{S}^{*}$ & $\lambda_{V}$ & $c_{v}\left(V^{(0)}-\lambda_{V}\right)$ & $\lambda_{A}$ & $c_{a}\left(\bar{A}-\lambda_{A}\right)$ & $\lambda_{H}$ & $c_{h}\left(\bar{H}-\lambda_{H}\right)$ \\
\hline$F_{0}^{* *}$ & $X_{0}^{* *}$ & $\lambda_{S}$ & $X_{S}^{* *}$ & $V^{(0) *}$ & 0 & $A^{(0) *}$ & 0 & $H^{(0) *}$ & 0 \\
\hline$F_{H}^{* *}$ & $X_{0}^{* *}$ & $\lambda_{S}$ & $X_{S}^{* *}$ & $V^{(0) *}$ & 0 & $A^{(0) *}$ & 0 & $\lambda_{H}$ & $c_{h}\left(H^{(0) *}-\lambda_{H}\right)$ \\
\hline$F_{A}^{* *}$ & $X_{0}^{* *}$ & $\lambda_{S}$ & $X_{S}^{* *}$ & $V^{(0) *}$ & 0 & $\lambda_{A}$ & $c_{a}\left(A^{(0) *}-\lambda_{A}\right)$ & $H^{(0) *}$ & 0 \\
\hline$F_{A H}^{* * *}$ & $X_{0}^{* *}$ & $\lambda_{S}$ & $X_{S}^{* *}$ & $V^{(0) *}$ & 0 & $\lambda_{A}$ & $c_{a}\left(A^{(0) *}-\lambda_{A}\right)$ & $\lambda_{H}$ & $c_{h}\left(H^{(0) *}-\lambda_{H}\right)$ \\
\hline$F_{V}^{* *}$ & $X_{0}^{* *}$ & $\lambda_{S}$ & $X_{S}^{* *}$ & $\lambda_{V}$ & $c_{v}\left(V^{(0) *}-\lambda_{V}\right)$ & $\overline{A^{*}}$ & 0 & $\overline{H^{*}}$ & 0 \\
\hline$F_{V H}^{* *}$ & $X_{0}^{* *}$ & $\lambda_{S}$ & $X_{S}^{* *}$ & $\lambda_{V}$ & $c_{v}\left(V^{(0) *}-\lambda_{V}\right)$ & $\overline{A^{*}}$ & 0 & $\lambda_{H}$ & $c_{h}\left(\overline{H^{*}}-\lambda_{H}\right)$ \\
\hline$F_{V A}^{* *}$ & $X_{0}^{* *}$ & $\lambda_{S}$ & $X_{S}^{* *}$ & $\lambda_{V}$ & $c_{v}\left(V^{(0) *}-\lambda_{V}\right)$ & $\lambda_{A}$ & $c_{a}\left(\overline{A^{*}}-\lambda_{A}\right)$ & $\overline{H^{*}}$ & 0 \\
\hline$F^{* *}$ & $X_{0}^{* *}$ & $\lambda_{S}$ & $X_{S}^{* *}$ & $\lambda_{V}$ & $c_{v}\left(V^{(0) *}-\lambda_{V}\right)$ & $\lambda_{A}$ & $c_{a}\left(\overline{A^{*}}-\lambda_{A}\right)$ & $\lambda_{H}$ & $c_{h}\left(\overline{H^{*}}-\lambda_{H}\right)$ \\
\hline
\end{tabular}

Tableau 14. Les équilibres du système (1) avec hydrolyse microbien où $r_{0}=g_{0}\left(X_{0}\right) X_{S}$, voir (9), (10), (11), (12) et (13). 
$X_{0}^{*}$ et $X_{0}^{* *}$ sont les solutions de l'équation $\zeta\left(X_{0}\right)=\delta\left(X_{0}\right)$, quand elles existent et :

$$
\begin{gathered}
X_{S}^{*}=\delta\left(X_{0}^{*}\right) \text { et } X_{S}^{* *}=\delta\left(X_{0}^{* *}\right) . \\
V^{(0)}=\gamma_{s v} X_{S}^{*}, A^{(0)}=\gamma_{s a} X_{S}^{*} \text { et } H^{(0)}=\gamma_{s h} X_{S}^{*} . \\
\bar{A}=A^{(0)}+\gamma_{v a} c_{v}\left(V^{(0)}-\lambda_{V}\right) \text { et } \bar{H}=H^{(0)}+\gamma_{v h} c_{v}\left(V^{(0)}-\lambda_{V}\right) . \\
V^{(0) *}=\gamma_{s v} X_{S}^{* *}, A^{(0) *}=\gamma_{s a} X_{S}^{* *} \text { et } H^{(0) *}=\gamma_{s h} X_{S}^{* *} . \\
\overline{A^{*}}=A^{(0) *}+\gamma_{v a} c_{v}\left(V^{(0) *}-\lambda_{V}\right) \text { et } \overline{H^{*}}=H^{(0) *}+\gamma_{v h} c_{v}\left(V^{(0) *}-\lambda_{V}\right) .
\end{gathered}
$$

3.2.1.1 Le cas $g_{0}\left(X_{0 i n}\right) \leqslant \frac{D}{c_{s} k_{0}}$.

On déduit les conditions d'existence des équilibres du modèle (1) à partir de la proposition 3.3 et de la proposition 2.3.

Proposition 3.7. Sous les hypothèses (H1) - (H4) et (H8), les conditions d'existence des équilibres du système (1) sont données par :

\begin{tabular}{|c||c|}
\hline L'équilibre & Conditions d'existence \\
\hline \hline$F_{l}$ & Existe toujours \\
\hline$F_{0}^{*}$ & $S_{i n}>\lambda_{S}$ \\
\hline$F_{H}^{*}$ & $S_{i n}>\lambda_{S}$ et $H^{(0)}>\lambda_{H}$ \\
\hline$F_{A}^{*}$ & $S_{i n}>\lambda_{S}$ et $A^{(0)}>\lambda_{A}$ \\
\hline$F_{A H}^{*}$ & $S_{i n}>\lambda_{S}, A^{(0)}>\lambda_{A}$ et $H^{(0)}>\lambda_{H}$ \\
\hline$F_{V}^{*}$ & $S_{i n}>\lambda_{S}$ et $V^{(0)}>\lambda_{V}$ \\
\hline$F_{V H}^{*}$ & $S_{i n}>\lambda_{S}, \bar{H}>\lambda_{H}$ et $V^{(0)}>\lambda_{V}$ \\
\hline$F_{V A}^{*}$ & $S_{i n}>\lambda_{S}, \bar{A}>\lambda_{A}$ et $V^{(0)}>\lambda_{V}$ \\
\hline$F^{*}$ & $S_{i n}>\lambda_{S}, \bar{H}>\lambda_{H}, \bar{A}>\lambda_{A}$ et $V^{(0)}>\lambda_{V}$ \\
\hline
\end{tabular}

Tableau 15. Les conditions d'existence des équilibres de (1), dans le cas $g_{0}\left(X_{0 i n}\right) \leqslant \frac{D}{c_{s} k_{0}}$.

On déduit les conditions de stabilité locale des équilibres du modèle (1) à partir de la proposition 3.3 et de la proposition 2.3 .

Proposition 3.8. Sous les hypothèses (H1) - (H4) et (H8), les conditions de stabilité des équilibres du système (1) sont données par :

\begin{tabular}{|c||c|}
\hline L'équilibre & Conditions de stabilité locale \\
\hline \hline$F_{l}$ & $S_{i n}<\lambda_{S}$ \\
\hline$F_{0}^{*}$ & $A^{(0)}<\lambda_{A}, H^{(0)}<\lambda_{H}$ et $V^{(0)}<\lambda_{V}$ \\
\hline$F_{H}^{*}$ & $A^{(0)}<\lambda_{A}$ et $V^{(0)}<\lambda_{V}$ \\
\hline$F_{A}^{*}$ & $H^{(0)}<\lambda_{H}$ et $V^{(0)}<\lambda_{V}$ \\
\hline$F_{A H}^{*}$ & $V^{(0)}<\lambda_{V}$ \\
\hline$F_{V}^{*}$ & $\bar{A}<\lambda_{A}$ et $\bar{H}<\lambda_{H}$ \\
\hline$F_{V H}^{*}$ & $\bar{A}<\lambda_{A}$ \\
\hline$F_{V A}^{*}$ & $\bar{H}<\lambda_{H}$ \\
\hline$F^{*}$ & dès qu'il existe \\
\hline
\end{tabular}

Tableau 16. Les conditions de stabilité des équilibres de (1), dans le cas $g_{0}\left(X_{0 i n}\right) \leqslant \frac{D}{c_{s} k_{0}}$ 
3.2.1.2 Le cas $g_{0}\left(X_{0 i n}\right)>\frac{D}{c_{s} k_{0}}$

On déduit les conditions d'existence et de stabilité locale des équilibres du modèle (1) à partir de la proposition 3.5 et de la proposition 2.3.

Proposition 3.9. Sous les hypothèses (H1) - (H4) et (H8), les conditions d'existence des équilibres du système (1) sont données par :

\begin{tabular}{|c||c|}
\hline L'équilibre & Conditions d'existence \\
\hline \hline$F_{l}$ & Existe toujours \\
\hline$F_{0}^{*}$ & $S_{i n}>\max \left(0, \bar{S}_{i n}\right)$ \\
\hline$F_{H}^{*}$ & $S_{i n}>\max \left(0, \bar{S}_{i n}\right)$ et $H^{(0)}>\lambda_{H}$ \\
\hline$F_{A}^{*}$ & $S_{i n}>\max \left(0, \bar{S}_{i n}\right)$ et $A^{(0)}>\lambda_{A}$ \\
\hline$F_{A H}^{*}$ & $S_{i n}>\max \left(0, \bar{S}_{i n}\right), A^{(0)}>\lambda_{A}$ et $H^{(0)}>\lambda_{H}$ \\
\hline$F_{V}^{*}$ & $S_{i n}>\max \left(0, \bar{S}_{i n}\right)$ et $V^{(0)}>\lambda_{V}$ \\
\hline$F_{V H}^{*}$ & $S_{i n}>\max \left(0, \bar{S}_{i n}\right), \bar{H}>\lambda_{H}$ et $V^{(0)}>\lambda_{V}$ \\
\hline$F_{V A}^{*}$ & $S_{i n}>\max \left(0, \bar{S}_{i n}\right), \bar{A}>\lambda_{A}$ et $V^{(0)}>\lambda_{V}$ \\
\hline$F^{*}$ & $S_{i n}>\max \left(0, \bar{S}_{i n}\right), \bar{H}>\lambda_{H}, \bar{A}>\lambda_{A}$ et $V^{(0)}>\lambda_{V}$ \\
\hline$F_{0}^{* *}$ & $\max \left(0, \bar{S}_{i n}\right)<S_{i n}<S_{i n}$ et $H^{(0) *}>\lambda_{H}$ \\
\hline$F_{H}^{* *}$ & $\max \left(0, \bar{S}_{i n}\right)<S_{i n}<\lambda_{S}$ et $A^{(0) *}>\lambda_{A}$ \\
\hline$F_{A}^{* * *}$ & $\max \left(0, \bar{S}_{i n}\right)<S_{i n}<\lambda_{S}, A^{(0) *}>\lambda_{A}$ et $H^{(0) *}>\lambda_{H}$ \\
\hline$F_{A H}^{* *}$ & $\max \left(0, \bar{S}_{i n}\right)<S_{i n}<\lambda_{S}$ et $V^{(0) *}>\lambda_{V}$ \\
\hline$F_{V}^{* *}$ & $\max \left(0, \bar{S}_{i n}\right)<S_{i n}<\lambda_{S}, \bar{H}^{*}>\lambda_{H}$ et $V^{(0) *}>\lambda_{V}$ \\
\hline$F_{V H}^{* *}$ & $\max \left(0, \bar{S}_{i n}\right)<S_{i n}<\lambda_{S}, \bar{A}^{*}>\lambda_{A}$ et $V^{(0) *}>\lambda_{V}$ \\
\hline$F_{V A}^{* *}$ & $\max \left(0, \bar{S}_{i n}\right)<S_{i n}<\lambda_{S}, \bar{H}^{*}>\lambda_{H}, \bar{A}^{*}>\lambda_{A}$ et $V^{(0) *}>\lambda_{V}$ \\
\hline$F^{* *}$ &
\end{tabular}

Tableau 17. Les conditions d'existence des équilibres de (1), dans le cas $g_{0}\left(X_{0 i n}\right)>\frac{D}{c_{s} k_{0}}$.

Proposition 3.10. Sous les hypothèses (H1) - (H4) et (H8), les conditions de stabilité des équilibres du système (1) sont données par :

\begin{tabular}{|c||c|}
\hline L'équilibre & Conditions de stabilité locale \\
\hline \hline$F_{l}$ & $S_{i n}<\lambda_{S}$ \\
\hline$F_{0}^{*}$ & $V^{(0)}<\lambda_{V}, A^{(0)}<\lambda_{A}$ et $H^{(0)}<\lambda_{H}$ \\
\hline$F_{H}^{*}$ & $A^{(0)}<\lambda_{A}$ et $V^{(0)}<\lambda_{V}$ \\
\hline$F_{A}^{*}$ & $H^{(0)}<\lambda_{H}$ et $V^{(0)}<\lambda_{V}$ \\
\hline$F_{A H}^{*}$ & $V^{(0)}<\lambda_{V}$ \\
\hline$F_{V}^{*}$ & $\bar{A}<\lambda_{A}$ et $\bar{H}<\lambda_{H}$ \\
\hline$F_{V H}^{*}$ & $\bar{A}<\lambda_{A}$ \\
\hline$F_{V A}^{*}$ & $\bar{H}<\lambda_{H}$ \\
\hline$F^{*}$ & dès qu'il existe \\
\hline$F_{0}^{* *}, F_{H}^{* *}, F_{A}^{* *}, F_{A H}^{* *}$, & Instables \\
$F_{V}^{* *}, F_{V H}^{* *}, F_{V A}^{* *}$ et $F^{* *}$ &
\end{tabular}

Tableau 18. Les conditions de stabilité des équilibres de (1), dans le cas $g_{0}\left(X_{0 i n}\right)>\frac{D}{c_{s} k_{0}}$. 


\subsubsection{Diagrammes opératoires}

Nous étudions le comportement du système (1) dans le plan $\left(D, S_{i n}\right)$ pour $X_{0 i n}$ fixé. Soit $X_{0 i n}=1$. Nous nous plaçons dans le cas où $g_{0}\left(X_{0 i n}\right) \leqslant \frac{D}{c_{s} k_{n}}$. Cette condition est équivalente à
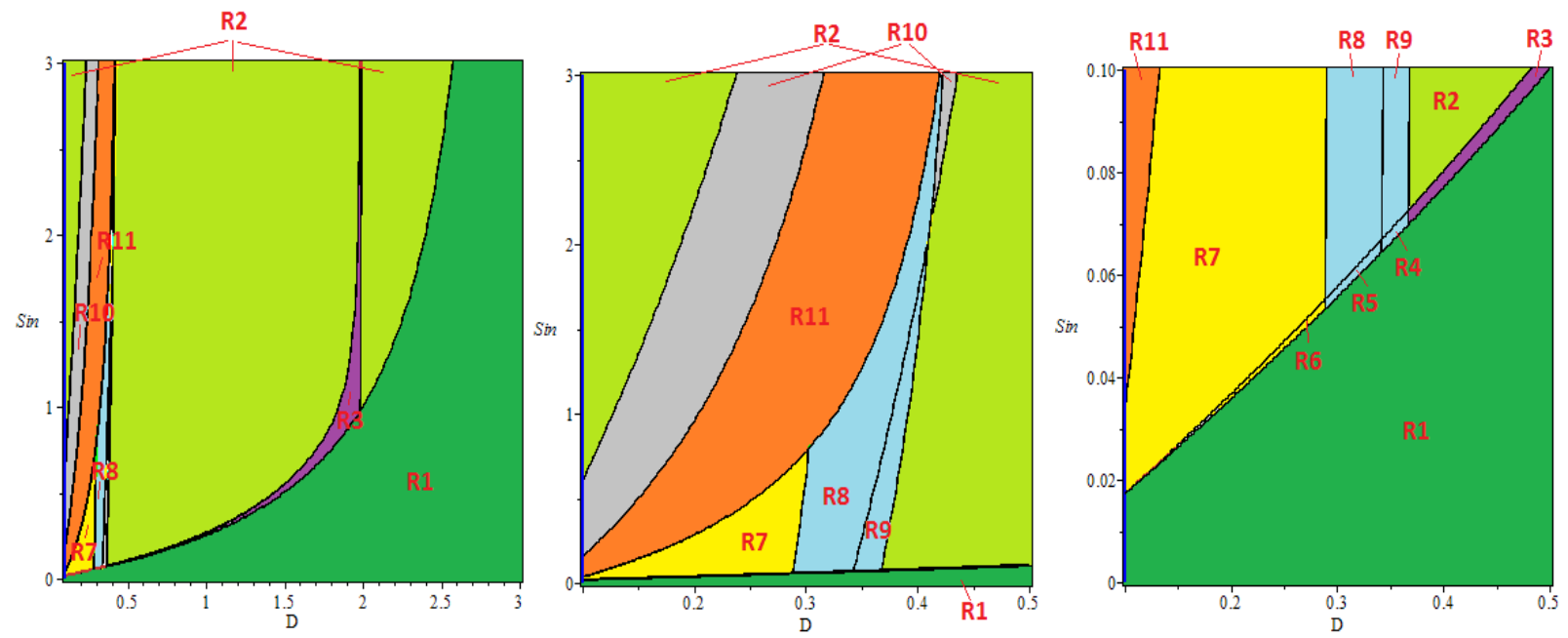

Figure 12. Diagramme opératoire pour $X_{0 i n}=1$ et $D \geqslant F_{01}$. (la figure au milieu est un agrandissement de la partie gauche de la figure à gauche et la figure à droite est un agrandissement du bas de la figure au milieu)

Pour le jeu de paramètres choisi dans [11], (Tableau A1), on a $F_{01}=0.1$. La figure 12 représente les diagrammes opératoires dans le plan $\left(D, S_{i n}\right)$, pour $X_{0 i n}=1$. Pour $X_{0 i n}=0$, on obtient les diagrammes opératoires du modèle avec hydrolyse enzymatique sans inhibition, voir Figure 2 à gauche.

Les régions $R_{10}, R_{4} \cup R_{5} \cup R_{8} \cup R_{9}$ et $R_{11}$ sont, respectivement, les régions de stabilité des équilibres $F_{A}^{*}$ (extinction des bactéries acétogènes et méthanogènes hydrogénotrophes), $F_{V H}^{*}$ (extinction des bactéries méthanogènes acétoclastes) et $F_{V A}^{*}$ (extinction des bactéries méthanogènes hydrogénotrophes), alors que les équilibres $E_{A}, E_{V H}$ et $E_{V A}$ du modèle avec hydrolyse enzymatique sont instables. De même, l'équilibre $F_{A H}^{*}$ de l'extinction des bactéries acétogènes existe et est instable dans $R_{5} \cup R_{6}$, il correspond à l'équilibre $E_{A H}$ qui peut être stable.

Pour $l=S, V, A$ et $H$, on prend les fonctions de croissance $g_{l}$ de la section 2.1 et $g_{0}\left(X_{0}\right)=\frac{m_{00} X_{0}}{k_{00}+X_{0}}$, avec $m_{00}=2.5$ et $K_{00}=1.5$.

Soient les fonctions $F_{L S}, F_{V 01}, F_{V 02}, F_{H 01}, F_{H 02}, F_{A 01}, F_{A 02}, F_{H 1}, F_{H 2}, F_{A 1}$ et $F_{A 2}$ définies par :

$$
\begin{array}{ll}
S_{i n}=\lambda_{S} & \Leftrightarrow S_{i n}=F_{L S}(D), \\
V^{(0)}=\lambda_{V} & \Leftrightarrow S_{i n}=F_{V 02}(D) \text { ou } S_{i n}=F_{V 01}(D), \\
H^{(0)}=\lambda_{H} & \Leftrightarrow S_{i n}=F_{H 02}(D) \text { ou } S_{i n}=F_{H 01}(D), \\
A^{(0)}=\lambda_{A} & \Leftrightarrow S_{i n}=F_{A 02}(D) \text { ou } S_{i n}=F_{A 01}(D), \\
\bar{H}=\lambda_{H} & \Leftrightarrow S_{i n}=F_{H 1}(D) \text { ou } S_{i n}=F_{H 2}(D), \\
\bar{A}=\lambda_{A} & \Leftrightarrow S_{i n}=F_{A 1}(D) \text { ou } S_{i n}=F_{A 2}(D),
\end{array}
$$


Ces fonctions vérifient alors les inégalités suivantes :

$$
\begin{array}{ll}
S_{i n}>\lambda_{S} & \Leftrightarrow S_{i n}>F_{L S}(D), \\
V^{(0)}>\lambda_{V} & \Leftrightarrow F_{V 02}(D)<S_{i n}<F_{V 01}(D), \\
H^{(0)}>\lambda_{H} & \Leftrightarrow F_{H 02}(D)<S_{i n}<F_{H 01}(D), \\
A^{(0)}>\lambda_{A} & \Leftrightarrow F_{A 02}(D)<S_{i n}<F_{A 01}(D), \\
\bar{H}>\lambda_{H} & \Leftrightarrow F_{H 1}(D)<S_{i n}<F_{H 2}(D), \\
\bar{A}>\lambda_{A} & \Leftrightarrow F_{A 1}(D)<S_{i n}<F_{A 2}(D),
\end{array}
$$

\begin{tabular}{|c|c|c|c|c|c|c|c|c|c|c|}
\hline Condition & Région & $F_{l}$ & $F_{0}^{*}$ & $F_{H}^{*}$ & $F_{A}^{*}$ & $F_{A H}^{*}$ & $F_{V}^{*}$ & $F_{V H}^{*}$ & $F_{V A}^{*}$ & $F_{*}$ \\
\hline$S_{i n}<F_{L S}(D)$ & $R_{1}$ & $\mathrm{~S}$ & & & & & & & & \\
\hline $\begin{array}{c}\quad\left(S_{i n}>F_{L S}(D) \text { et } S_{i n}<F_{H 02}(D)\right) \\
\text { ou }\left(S_{i n}>F_{H 01}(D), S_{i n}<F_{A 02}(D)\right. \\
\left.\text { et } S_{i n}<F_{V 02}(D)\right) \text { ou }\left(S_{i n}>F_{A 01}(D)\right)\end{array}$ & $R_{2}$ & I & $\mathrm{S}$ & & & & & & & \\
\hline $\begin{array}{l}S_{i n}>F_{L S}(D), S_{i n}<F_{V 02}(D) \\
\text { et } F_{H 02}(D)<S_{i n}<F_{H 01}(D)\end{array}$ & $R_{3}$ & I & I & S & & & & & & \\
\hline $\begin{array}{c}S_{i n}>F_{L S}(D), S_{i n}>F_{V 02}(D), \\
S_{i n}<F_{H 01}(D) \text { et } S_{i n}<F_{A 02}(D)\end{array}$ & $R_{4}$ & I & I & I & & & I & S & & \\
\hline $\begin{array}{c}S_{i n}>F_{L S}(D), S_{i n}<F_{H 01}(D) \\
S_{i n}>F_{A 02}(D) \text { et } S_{i n}<F_{A 1}(D)\end{array}$ & $R_{5}$ & I & I & I & I & I & I & $\mathrm{S}$ & & \\
\hline $\begin{array}{c}S_{i n}>F_{L S}(D) \\
S_{i n}>F_{A 1}(D) \text { et } S_{i n}<F_{H 01}(D)\end{array}$ & $R_{6}$ & I & I & I & I & I & I & I & I & $\mathrm{S}$ \\
\hline $\begin{array}{c}S_{i n}>F_{H 01}(D) \\
S_{i n}>F_{A 1}(D) \text { et } S_{i n}<F_{H 2}(D)\end{array}$ & $R_{7}$ & I & I & & I & & I & I & I & $\mathrm{S}$ \\
\hline $\begin{array}{c}S_{i n}>F_{V 02}(D), S_{i n}>F_{A 02}(D) \\
S_{i n}>F_{H 01}(D), S_{i n}<F_{A 1}(D) \\
\quad \text { et } S_{i n}<F_{H 2}(D)\end{array}$ & $R_{8}$ & $\mathrm{I}$ & I & & I & & I & $\mathrm{S}$ & & \\
\hline $\begin{array}{c}S_{i n}>F_{V 02}(D) \\
F_{H 01}(D)<S_{i n} \text { et } S_{i n}<F_{A 02}(D)\end{array}$ & $R_{9}$ & I & I & & & & I & $\mathrm{S}$ & & \\
\hline $\begin{array}{c}\left(S_{i n}>F_{A 02}(D) \text { et } S_{i n}<F_{V 02}(D)\right) \\
\text { ou }\left(F_{V 01}(D)<S_{i n} \text { et } S_{i n}<F_{A 01}(D)\right)\end{array}$ & $R_{10}$ & I & I & & $\mathrm{S}$ & & & & & \\
\hline$S_{i n}>F_{H 2}(D)$ et $S_{i n}<F_{V 01}(D)$ & $R_{11}$ & $\mathrm{I}$ & $\mathrm{I}$ & & I & & $\mathrm{I}$ & & $\mathrm{S}$ & \\
\hline
\end{tabular}

En utilisant la proposition 3.7 et la proposition 3.8, on a le résultat suivant :

Proposition 3.11. Le plan $\left(D, S_{i n}\right)$ est divisé en onze régions décrites dans le tableau 19 :

Tableau 19. Existence et stabilité des équilibres du système (1) selon $\left(D, S_{i n}\right)$, avec $D \geqslant$ $F_{01}$

Ici, la lettre $S$ (resp. $I$ ) signifie que l'équilibre correspondant est asymptotiquement stable (resp. instable). L'absence de lettre signifie que l'équilibre n'existe pas.

D'après ce qui précède, nous avons mis en évidence un comportement asymptotique des équilibres différent, selon la modélisation de l'hydrolyse enzymatique ou par un compartiment hydrolytique microbien. L'hydrolyse affecte la nature et les zones de stabilité des équilibres.

\subsubsection{Taux de biogaz produit}

\subsubsection{Taux de méthane produit}

Le taux de méthane produit est donné par la formule suivante :

$$
Q_{C H_{4}}=\alpha_{1} g_{A}\left(A_{\left.\right|_{A=A^{*}}}\right) X_{\left.A\right|_{X_{A}=X_{A}^{*}}}+\alpha_{2} g_{H}\left(H_{\left.\right|_{H=H^{*}}}\right) X_{\left.H\right|_{X_{H}=X_{H}^{*}}}
$$


avec $\alpha_{1}=\frac{1-c_{a}}{c_{a}}$ et $\alpha_{2}=\frac{1-c_{h}}{c_{h}}$.

En se plaçant dans le cas où $g_{0}\left(X_{0 i n}\right) \leqslant \frac{D}{c_{s} k_{0}}$. Le taux de méthane produit, pour chaque équilibre, est donnée dans le tableau 20 :

\begin{tabular}{|c||c|}
\hline Équilibre & $Q_{C H_{4}}$ \\
\hline \hline$F_{l}^{*}, F_{0}^{*}$ et $F_{V}^{*}$ & 0 \\
\hline$F_{H}^{*}$ & $\alpha_{2} c_{h} D\left(H^{(0)}-\lambda_{H}\right)$ \\
\hline$F_{A}^{*}$ & $\alpha_{1} c_{a} D\left(A^{(0)}-\lambda_{A}\right)$ \\
\hline$F_{A H}^{*}$ & $\alpha_{1} c_{a} D\left(A^{(0)}-\lambda_{A}\right)+\alpha_{2} c_{h} D\left(H^{(0)}-\lambda_{H}\right)$ \\
\hline$F_{V H}^{*}$ & $\alpha_{2} c_{h} D\left(\bar{H}-\lambda_{H}\right)$ \\
\hline$F_{V A}^{*}$ & $\alpha_{1} c_{a} D\left(\bar{A}-\lambda_{A}\right)$ \\
\hline$F^{*}$ & $\alpha_{1} c_{a} D\left(\bar{A}-\lambda_{A}\right)+\alpha_{2} c_{h} D\left(\bar{H}-\lambda_{H}\right)$ \\
\hline
\end{tabular}

Tableau 20. Taux de méthane produit pour chaque équilibre du modèle avec hydrolyse microbien

On rappelle que $\bar{A}=A^{(0)}+\gamma_{v a} c_{v}\left(V^{(0)}-\lambda_{V}\right), \bar{H}=H^{(0)}+\gamma_{v h} c_{v}\left(V^{(0)}-\lambda_{V}\right)$, $V^{(0)}=\gamma_{s v} X_{S}^{*}, A^{(0)}=\gamma_{s a} X_{S}^{*}, H^{(0)}=\gamma_{s h} X_{S}^{*}, X_{S}^{*}=\delta\left(X_{0}^{*}\right)$ et $X_{0}^{*}$ est la solution de $\zeta\left(X_{0}\right)=\delta\left(X_{0}\right)$.

1) Dans le cas où $V^{(0)}<\lambda_{V}$ : les équilibres $F_{V H}^{*}, F_{V A}^{*}$ et $F^{*}$ n'existent pas et on a $\bar{A}<A^{(0)}$ et $\bar{H}<H^{(0)}$. Par suite, le taux maximal de méthane est donné soit par l'équilibre $F_{A H}^{*}$, soit par $F_{H}^{*}$, soit par $F_{A}^{*}$.

Si $H^{(0)}>\lambda_{H}$ et $A^{(0)}>\lambda_{A}$ alors le taux maximal de méthane est donné par $F_{A H}^{*}$. Notons que si ces conditions sont vérifiées alors $F_{A H}^{*}$ est stable.

Si $H^{(0)}>\lambda_{H}$ et $A^{(0)}<\lambda_{A}$ alors le taux maximal de méthane est donné par $F_{H}^{*}$. Ces conditions sont vérifiées pour $\left(D, S_{i n}\right)$ dans la région $R_{3}$.

Si $H^{(0)}<\lambda_{H}$ et $A^{(0)}>\lambda_{A}$ alors le taux maximal de méthane est donné par $F_{A}^{*}$. Ces conditions sont vérifiées pour $\left(D, S_{i n}\right)$ dans la région $R_{10}$.

2) Dans le cas où $V^{(0)}>\lambda_{V}$, on a $\bar{A}>A^{(0)}$ et $\bar{H}>H^{(0)}$. Le taux maximal de méthane est alors donné soit par l'équilibre $F_{V H}^{*}$, soit par $F_{V A}^{*}$, soit par $F^{*}$.

Si $\bar{H}>\lambda_{H}$ et $\bar{A}>\lambda_{A}$ alors le taux maximal de méthane est donné par $F^{*}$. Ces conditions sont vérifiées pour $\left(D, S_{i n}\right)$ dans les régions $R_{6}$ ou $R_{7}$ où $F^{*}$ est stable.

Si $V^{(0)}>\lambda_{V}, \bar{H}<\lambda_{H}$ et $\bar{A}>\lambda_{A}$ alors le taux maximal de méthane est donnée par $F_{V A}^{*}$. Ces conditions sont vérifiées dans la région $R_{11}$.

Si $V^{(0)}>\lambda_{V}, \bar{H}>\lambda_{H}$ et $\bar{A}<\lambda_{A}$ alors le taux maximal de méthane est donnée par $F_{V H}^{*}$. Ces conditions sont vérifiées dans les régions $R_{4}, R_{5}, R_{8}$ ou $R_{9}$, où $F_{V H}^{*}$ est stable.

On représente, dans la figure 13, le taux maximal du méthane produit en fonction de la dilution $D$. On fait varier la valeur de la concentration du substrat sous forme particulaire $X_{0 i n}$ et la valeur de la concentration du substrat sous forme soluble $S_{i n}$ de telle manière à maintenir la somme constante.

En maintenant la somme $X_{0 i n}+S_{i n}$ constante, le taux de méthane est plus élevé avec le substrat soluble qu'avec le substrat solide. 


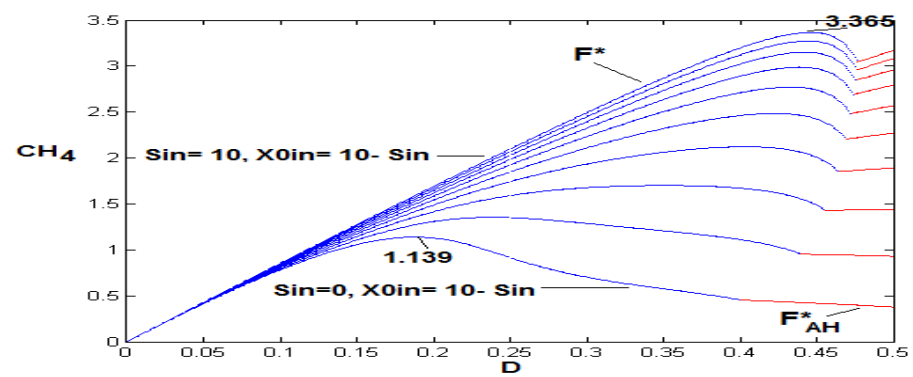

Figure 13. La variation du taux de méthane pour $S_{i n}+X_{0 i n}=10$, pour le modèle avec compartiment hydrolytique microbien

\subsubsection{Taux d'hydrogène produit}

Le taux d'hydrogène produit est donné par la formule suivante :

$$
Q_{H 2}=\alpha_{3} g_{S}\left(S_{\left.\right|_{S=S^{*}}}\right) X_{\left.S\right|_{X_{S}=X_{S}^{*}}}+\alpha_{4} g_{V}\left(V_{\left.\right|_{V=V^{*}}}\right) X_{\left.V\right|_{X_{V}=X_{V}^{*}}}
$$

avec $\alpha_{3}=\frac{1-c_{s}}{c_{s}}$ et $\alpha_{4}=\frac{1-c_{v}}{c_{v}}$. Nous nous plaçons dans le cas où $g_{0}\left(X_{0 i n}\right) \leqslant \frac{D}{c_{s} k_{0}}$. Le tableau 21 donne le taux d'hydrogène produit en chaque équilibre.

\begin{tabular}{|c||c|}
\hline Équilibre & $Q_{H_{2}}$ \\
\hline \hline$F_{l}$ & 0 \\
\hline$F_{0}^{*}, F_{H}^{*}, F_{A}^{*}$ et $F_{A H}^{*}$ & $Q_{H_{21}}:=\alpha_{3} D X_{S}^{*}$ \\
\hline$F_{V}^{*}, F_{V H}^{*}, F_{V A}^{*}$ et $F^{*}$ & $Q_{H_{22}}:=\alpha_{3} D X_{S}^{*}+\alpha_{4} D c_{v}\left(V^{(0)}-\lambda_{V}\right)$ \\
\hline
\end{tabular}

Tableau 21. Taux d'hydrogène produit pour chaque équilibre du modèle avec compartiment hydrolytique microbien

1) Dans le cas, $V^{(0)}<\lambda_{V}$, les équilibres $F_{V}^{*}, F_{V H}^{*}, F_{V A}^{*}$ et $F^{*}$ n'existent pas et on a $Q_{H_{21}}>Q_{H_{22}}$. Par suite, le taux maximal de l'hydrogène est donné soit par l'équilibre $F_{A H}^{*}$, soit par $F_{H}^{*}$, soit par $F_{A}^{*}$, soit par $F_{0}^{*}$.

Si $S^{\text {in }}>\lambda_{S}$ alors le taux maximal d'hydrogène est donné par $F_{0}^{*}$. Cet équilibre est stable dans la région $R_{2}$.

Si $H^{(0)}>\lambda_{H}$ alors le taux maximal d'hydrogène est donné par $F_{H}^{*}$. Ces conditions sont vérifiées dans la région $R_{3}$ où $F_{H}^{*}$ est stable.

$\mathrm{Si} A^{(0)}>\lambda_{A}$ alors le taux maximal d'hydrogène est donné par $F_{A}^{*}$ qui est stable dans la région $R_{10}$.

Si $H^{(0)}>\lambda_{H}$ et $A^{(0)}>\lambda_{A}$ alors le taux maximal d'hydrogène est donné par $F_{A H}^{*}$. Notons que si ces conditions sont vérifiées alors $F_{A H}^{*}$ est stable.

2) Dans le cas, $V^{(0)}>\lambda_{V}, Q_{H_{22}}>Q_{H_{21}}$. Par suite, le taux maximal d'hydrogène est donné soit par l'équilibre $F_{V H}^{*}$, soit par $F_{V A}^{*}$, soit par $F_{V}^{*}$, soit par $F^{*}$.

Si $\bar{H}>\lambda_{H}$ et $\bar{A}>\lambda_{A}$ alors $F^{*}$ existe et le taux maximal d'hydrogèneest donnée par cet équilibre. $F^{*}$ est stable dans les régions $R_{6}$ ou $R_{7}$.

Si $\bar{A}>\lambda_{A}$ alors $F_{V A}^{*}$ existe et le taux maximal d'hydrogène est donnée par cet équilibre. Cet équilibre est stable dans la région $R_{11}$ c'est à dire pour $\bar{H}<\lambda_{H}$.

Si $\bar{H}>\lambda_{H}$ alors $F_{V H}^{*}$ existe et le taux maximal d'hydrogène est donnée par cet équilibre. Cet équilibre est stable dans la région $R_{4}, R_{5}, R_{8}$ ou $R_{9}$ c'est à dire pour $\bar{A}<\lambda_{A}$. 
Si $\bar{H}<\lambda_{H}$ et $\bar{A}<\lambda_{A}$ alors le taux maximal d'hydrogène est donnée par $F_{V}^{*}$. Notons que si ces conditions sont vérifiées alors $F_{V}^{*}$ est stable.

Le taux maximal d'hydrogène produit est représenté dans la figure 14. Le taux d'hydrogène est plus élevé avec le substrat soluble qu'avec le substrat solide, $X_{0 i n}+S_{i n}$ étant maintenue constante.

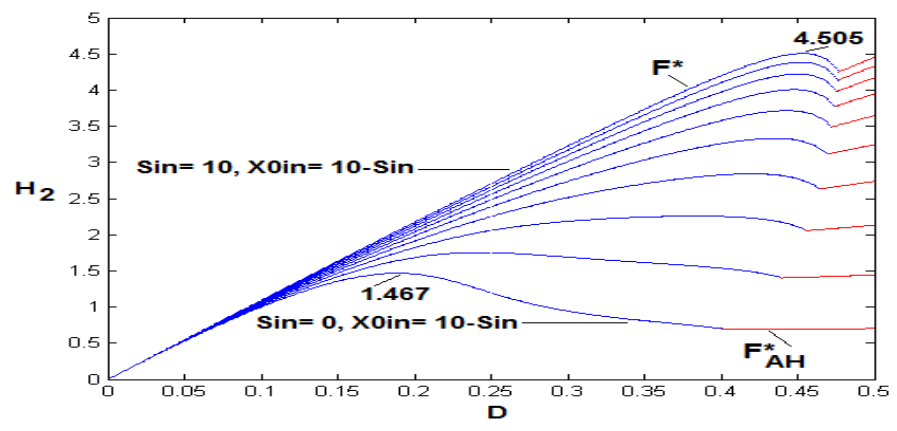

Figure 14. La variation du taux d'hydrogène pour $S_{i n}+X_{0 i n}=10$, pour le modèle avec compartiment hydrolytique microbien.

\section{Discussion}

Nous avons étudié des modèles de digestion anaérobie à quatre étapes, avec dégradation du substrat sous forme particulaire. Nous avons analysé deux modèles tenant compte de l'étape d'hydrolyse. Cette phase est considérée dans le premier modèle comme une simple hydrolyse enzymatique, ce qui ajoute au système une variable, la concentration $X_{0}$ du substrat lentement biodégradable, dont la dynamique n'est pas couplée aux autres variables. Nous avons étudié ensuite le cas plus complexe d'un compartiment hydrolytique microbien où la dynamique de $X_{0}$ est couplée cette fois aux deux variables du sous-modèle (5), la concentration du substrat simple et celle des bactéries acidogènes. Nous avons mis en évidence, dans le modèle avec hydrolyse enzymatique, le rôle des inhibitions dans le système en étudiant dans un premier temps ce modèle sans inhibition puis en tenant compte des inhibitions dans les fonctions de croissance. Le modèle avec compartiment microbien hydrolytique a été analysé dans le cas sans inhibition.

Nous avons établi les conditions d'existence et le comportement asymptotique local ou global des points d'équilibre pour les modèles avec et sans inhibition. De plus, nous avons déterminé le taux de biogaz pour chaque équilibre pour les deux modèles et nous avons calculé le taux maximal de biogaz produit pour différentes valeurs des substrats à l'entrée.

Nous avons montré que prendre en compte de l'inhibition dans le modèle influe sur la stabilité des équilibres. Des zones de bistabilité apparaissent. Grâce aux diagrammes opératoires qui illustrent les zones d'existence, de stabilité et de bistabilité, nous avons montré comment les régions de stabilité peuvent s'élargir en fonction des paramètres opératoires, le taux de dilution $D$ et les substrats en entrée $S_{i n}$ et $X_{0 i n}$. On pourra alors faire varier le taux de dilution pour éviter les régions de bistabilité. D'autre part, nous avons montré que la valeur des paramètres influe sur le taux de biogaz produit. L'augmentation des concentrations des deux substrats en entrée pour un modèle avec hydrolyse enzymatique des biomasses favorise bien l'augmentation du biogaz produit. Le modèle avec hydrolyse enzymatique sans inhibition produit plus de biogaz que le modèle avec inhibition. Pour des valeurs fixées des concentrations des substrats en entrée, il existe un taux de dilution qui maximise le taux de méthane. Pour ce taux, la concentration des bactéries méthanogènes acétoclastes augmente et celle de l'acétate devient assez faible, ce qui affaiblit l'inhibition des bactéries méthanogènes hydrogénotrophes qui sont les producteurs de méthane. Pour l'hydrogène produit, il existe un taux de dilution qui maximise le taux d'hydrogène, pour des concentrations des bactéries 
méthanogènes hydrogénotrophes très faibles.

En passant du modèle avec hydrolyse enzymatique au modèle avec compartiment hydrolytique microbien, l'équilibre de l'extinction des acétogènes qui était stable devient instable et les équilibres correspondant à l'extinction des acétogènes et des méthanogènes hydrogénotrophes, ou à l'extinction des méthanogènes acétoclastes, ou à l'extinction des méthanogènes hydrogénotrophes qui étaient instables deviennent stables. Nous avons montré que le comportement du système dépend de la modélisation de l'étape d'hydrolyse choisie. Selon les conditions d'existence et de stabilité, nous avons montré que le taux maximal de méthane et d'hydrogène produit est atteint en un équilibre stable. Ces taux sont explicitement déterminés, selon les paramètres des modèles considérés.

\section{Conclusion}

L'objectif principal, dans ce travail, est l'étude de modèles de la digestion anaérobie à quatre étapes pour l'optimisation de la production de méthane et d'hydrogène. Ce processus biologique de conversion de la matière organique en mélange gazeux, est important à la fois pour le traitement des eaux usées et pour la production du biogaz. L'étude des équilibres de ces modèles et de leur stabilité en fonction des paramètres à l'entrée du processus permet de déterminer les produits en sortie, ainsi que les quantités de gaz pouvant être produites. Cette étude a montré que les valeurs des paramètres ainsi que la présence de l'inhibition de certaines espèces microbiennes dans le système influent sur le taux de biogaz. La production du biogaz diffère suivant le choix de la modélisation de l'hydrolyse. Les résultats obtenus permettront potentiellement de proposer de nouvelles options aux biologistes pour déterminer des conditions opératoires optimisant la production de biogaz. En perspective, on pourra étudier le taux de biogaz produit pour le modèle avec hydrolyse microbienne en prenant en compte l'inhibition des fonctions de croissance.

\section{Annexe}

\section{Preuve de la Proposition 2.3}

En appliquant la même technique que [11, Lemme 3.1], on peut déduire un résultat de stabilité globale des équilibres du système (1). L'énoncé du Lemme 3.1 de [11] est le suivant :

\section{Lemme A.1 :}

On suppose que $F: \mathbb{R}^{\mathrm{m}} \longrightarrow \mathbb{R}^{\mathrm{m}}$ est de classe $\mathcal{C}^{1}$ et que l'équation $\frac{d Y}{d t}=F(Y)$ possède un équilibre globalement asymptotiquement stable $Y^{*}$. On suppose que $g:[0,+\infty[\longrightarrow[0,+\infty[$ est de classe $\mathcal{C}^{1}$, qu'elle est croissante et que $g(0)=0$. Pour des valeurs fixées de $D>0$ et $c>0$, soit $\lambda$ la solution de l'équation $g(S)=D$ et soit $f: \mathbb{R}^{\mathrm{m}} \longrightarrow\left[0,+\infty\left[\right.\right.$ de classe $\mathcal{C}^{1}$.

On considère le système :

$$
\left\{\begin{array}{l}
\frac{d Y}{d t}=F(Y) \\
\frac{d S}{d t}=-D S+f(Y)-\frac{1}{c_{s}} g(S) X \\
\frac{d X}{d t}=-D X+g(S) X
\end{array}\right.
$$

avec $S(0)>0$ et $X(0)>0$. On a,

1) $\operatorname{Si} \lambda>\frac{f\left(Y^{*}\right)}{D}$, alors toutes les solutions convergent vers $\left(Y^{*}, \frac{f\left(Y^{*}\right)}{D}, 0\right)$.

2) Si $0<\lambda<\frac{f\left(Y^{*}\right)}{D}$, alors toutes les solutions convergent vers $\left(Y^{*}, \lambda, X^{*}\right)$, avec $X^{*}=$ $c\left(\frac{f\left(Y^{*}\right)}{D}-\lambda\right)$. 
Nous utilisons le Lemme A.1 pour démontrer la proposition 2.3. En effet, le système (1) possède une structure en cascade donc on pourra étudier dans un premier temps le sous-système suivant :

$$
\left\{\begin{array}{l}
\frac{d X_{0}}{d t}=D\left(X_{0 i n}-X_{0}\right)-k_{h y d} X_{0} \\
\frac{d S}{d t}=D\left(S_{i n}-S\right)-\frac{1}{c_{s}} g_{S}(S) X_{S}+f\left(X_{0}\right) \\
\frac{d X_{S}}{d t}=\left(g_{S}(S)-D\right) X_{S}
\end{array}\right.
$$

avec $f\left(X_{0}\right)=k_{0} k_{h y d} X_{0}$.

La première équation de ce sous-système s'écrit $\frac{d X_{0}}{d t}=F\left(X_{0}\right)$, avec $\mathrm{F}$ une fonction de classe $\mathcal{C}^{1}$. Elle possède un équilibre globalement stable $X_{0}^{*} \stackrel{d t}{=} \frac{D}{D+k_{h y d}} X_{0 i n}$. De plus, $g_{S}:[0,+\infty[\longrightarrow$ $\left[0,+\infty\left[\right.\right.$ est de classe $\mathcal{C}^{1}$, croissante et $g(0)=0$, d'après $(\mathbf{H 1}) . \lambda_{S}$ est la solution de l'équation $g_{S}(S)=D$. La fonction $f$ de $\left[0,+\infty\left[\longrightarrow\left[0,+\infty\left[\right.\right.\right.\right.$ est de classe $\mathcal{C}^{1}$ et on a $S(0)>0$ et $X_{S}(0)>$ 0 . En appliquant le Lemme A.1, on déduit que :

- Si $\lambda_{S}>S_{i n}^{*}$ avec $S_{i n}^{*}=\left(\frac{f\left(X_{0}^{*}\right)}{D}+S_{i n}\right)$, alors toutes les solutions convergent vers $\left(X_{0}^{*}, S_{i n}^{*}, 0\right)$

- Si $0<\lambda_{S}<S_{i n}^{*}$, alors toutes les solutions convergent vers $\left(X_{0}^{*}, \lambda_{S}, X_{S}^{*}\right)$ avec $X_{S}^{*}=$ $c s\left(S_{i n}^{*}-\lambda_{S}\right)$.

On applique le Lemme A.1, en utilisant le résultat précédent sur les deux équations suivantes du système (1) et en éliminant les cas où $\lambda_{i}, i=A, V, H$, sont négatifs. De la même manière, on détermine les conditions de stabilité globale des équilibres du système (1). Les conditions d'existence et de stabilité globale des équilibres du système (1) se résument dans le tableau 2 de la proposition 2.3.

\section{Preuve de la Proposition 2.4}

On déduit le tableau 3 de la Proposition 2.4 en utilisant la Proposition 2.3, l'inégalité (3) et les inégalités suivantes :

- $S_{\text {in }}<F(D)$ est équivalent à $S_{i n}^{*}<\lambda_{S}$. Dans ce cas $\left(D, S_{i n}\right) \in R_{1}$.

- $S_{\text {in }}<F(D)+K_{H}(D)$ est équivalent à $H^{(0)}<\lambda_{H}$. Or $H^{(0)}<\lambda_{H}$ est équivalent à $g_{H}\left(H^{(0)}\right)<D$. D'après (3), on obtient $g_{V}\left(V^{(0)}\right)<g_{A}\left(A^{(0)}\right)<D$, ce qui donne $A^{(0)}<\lambda_{A}$ et $V^{(0)}<\lambda_{V}$. Dans ce cas $\left(D, S_{i n}\right) \in R_{1} \cup R_{2}$.

$-S_{\text {in }}<F(D)+K_{A}(D)$ est équivalent à $A^{(0)}<\lambda_{A}$. Or $A^{(0)}<\lambda_{A}$ est équivalent à $g_{A}\left(A^{(0)}\right)<D$. D'après (3), on obtient $g_{V}\left(V^{(0)}\right)<D$, ce qui donne $V^{(0)}<\lambda_{V}$. Dans ce cas $\left(D, S_{\text {in }}\right) \in R_{1} \cup R_{2} \cup R_{3}$.

$-S_{i n}>F(D)+K_{A}(D)$ est équivalent à $A^{(0)}>\lambda_{A}$. Or $A^{(0)}>\lambda_{A}$ est équivalent à $g_{A}\left(A^{(0)}\right)>D$. D'après (3), on obtient $g_{H}\left(H^{(0)}\right)>D$, ce qui donne $H^{(0)}>\lambda_{H}$. Dans ce cas $\left(D, S_{\text {in }}\right) \in R_{4} \cup R_{5}$.

$-S_{\text {in }}<F(D)+K_{V}(D)$ est équivalent à $V^{(0)}<\lambda_{V}$. Dans ce cas $\left(D, S_{i n}\right) \in R_{1} \cup R_{2} \cup$ $R_{3} \cup R_{4}$.

$-S_{\text {in }}>F(D)+K_{V}(D)$ est équivalent à $V^{(0)}>\lambda_{V}$. Or $V^{(0)}>\lambda_{V}$ est équivalent à $g_{V}\left(V^{(0)}\right)>D$. D'après (3), on obtient $D<g_{A}(\bar{A})<g_{H}(\bar{H})$. Or $D<g_{A}(\bar{A})$ est équivalent à $\lambda_{A}<\bar{A}$ et $D<g_{H}(\bar{H})$ est équivalent à $\lambda_{H}<\bar{H}$. Dans ce cas $\left(D, S_{i n}\right) \in R_{5}$.

\section{Preuve de la Proposition 2.5}

On déduit le tableau 4 de la Proposition 2.5 de la même manière que dans la preuve de la Proposition 2.4, en utilisant la Proposition 2.3, l'inégalité (3) et les inégalités suivantes 


$$
\begin{aligned}
& -X_{0 i n}<G(D) \text { est équivalent à } S_{i n}^{*}<\lambda_{S} . \\
& -X_{0 i n}<L_{H}(D)+G(D) \text { est équivalent à } H^{(0)}<\lambda_{H} . \\
& -X_{0 i n}<L_{A}(D)+G(D) \text { est équivalent à } A^{(0)}<\lambda_{A} . \\
& -X_{0 i n}<L_{V}(D)+G(D) \text { est équivalent à } V^{(0)}<\lambda_{V} .
\end{aligned}
$$

La preuve des résultats des tableaux 7, 8 et 9 est une simple adaptation de ceux de [11], voir section 4.1 de [11]. Pour l'étude de la stabilité locale du sous-modèle avec inhibition, on utilise la matrice Jacobienne de la page 73 de [11].

Les preuves de la Proposition 3.1, du Lemme 3.1, du Lemme 3.2, de la Proposition 3.2 et de la Proposition 3.4 sont données dans [2], voir preuve de la Proposition V.2, du Lemme V.1, du Lemme V.2, de la Proposition V.3 et de la Proposition V.4, de [2].

\section{Preuve de la Proposition 3.3}

Les conditions d'existence des équilibres sont données dans la Proposition 3.2. Pour étudier la stabilité locale de chaque équilibre, on détermine le signe de la partie réelle des valeurs propres de la matrice Jacobienne J.

La matrice Jacobienne associée à l'équilibre $F_{0}$ est

$$
J_{F_{0}}=\left[\begin{array}{ccc}
-D & 0 & -g_{0}\left(X_{0 i n}\right) \\
0 & -D & k_{0} g_{0}\left(X_{0 i n}\right)-\frac{1}{c_{s}} g_{S}\left(S_{i n}\right) \\
0 & 0 & g_{S}\left(S_{i n}\right)-D
\end{array}\right]
$$

Les valeurs propres de $J_{F_{0}}$ sont : $-D,-D$ et $g_{S}\left(S_{i n}\right)-D$. Par suite, $F_{0}$ est LES si et seulement si $g_{S}\left(S_{i n}\right)<D$, ce qui est équivalent à $S_{i n}<\lambda_{S}$.

La matrice Jacobienne associée à l'équilibre $F_{1}^{*}$ est :

$$
J_{F_{1}^{*}}=\left[\begin{array}{ccc}
-m_{11} & 0 & -m_{13} \\
m_{21} & -m_{22} & \theta \\
0 & m_{32} & 0
\end{array}\right]
$$

avec $m_{11}=D+\frac{d g_{0}}{d X_{0}}\left(X_{0}^{*}\right) X_{S}^{*}, m_{13}=g_{0}\left(X_{0}^{*}\right), m_{21}=k_{0} \frac{d g_{0}}{d X_{0}}\left(X_{0}^{*}\right) X_{S}^{*}$, $m_{22}=D+\frac{1}{c_{s}} \frac{d g_{S}}{d S}\left(\lambda_{S}\right) X_{S}^{*}, m_{32}=\frac{d g_{S}}{d S}\left(\lambda_{S}\right) X_{S}^{*}$ et $\theta=\frac{-D}{c_{s}}+k_{0} g_{0}\left(X_{0}^{*}\right)$.

Le polynôme caractéristique de $J_{F_{1}^{*}}$ est :

$$
P_{J_{F_{1}^{*}}}=-\lambda^{3}-\left(m_{11}+m_{22}\right) \lambda^{2}-\left(m_{11} m_{22}-\theta m_{32}\right) \lambda-m_{32}\left(-m_{11} \theta+m_{21} m_{13}\right) .
$$

On pose $c_{0}=-1, c_{1}=-\left(m_{11}+m_{22}\right), c_{2}=-\left(m_{11} m_{22}-\theta m_{32}\right)$ et $c_{3}=m_{32}\left(-m_{11} \theta+m_{21} m_{13}\right)$.

On a $c_{0}<0$ et $c_{1}<0$. De plus, $c_{2}$ et $c_{3}$ sont négatifs si $\zeta^{\prime}\left(X_{0}^{*}\right)<-c_{s} k_{0}$. En calculant $c_{1} c_{2}-c_{0} c_{3}$, on voit que $c_{1} c_{2}-c_{0} c_{3}>0$. Par suite, le critère de Routh-Hurwitz est satisfait si la condition $\zeta^{\prime}\left(X_{0}^{*}\right)<-c_{s} k_{0}$ est vérifiée. On conclut que l'équilibre $F_{1}^{*}$ est localement stable dès qu'il existe.

\section{Preuve de la Proposition 3.5}

Comme pour la Proposition 3.3, la stabilité locale de $F_{0}$ est donnée par le signe de la partie réelle des valeurs propres de la matrice Jacobienne $J_{F_{0}}$. Pour les deux autres équilibres, on utilise le critère de Routh-Hurwitz. Le critère de Routh-Hurwitz est satisfait si la condition $\zeta^{\prime}\left(X_{0}^{*}\right)<-c_{s} k_{0}$ est vérifiée, $F_{1}^{*}$ est donc LES. Or l'équilibre $F_{1}^{* *}$ vérifie $\zeta^{\prime}\left(X_{0}^{* *}\right)>-c_{s} k_{0}$ alors le critère de Routh-Hurwitz n'est pas satisfait. Par suite, $F_{1}^{* *}$ est instable. 
La preuve de la Proposition 3.6 est une simple adaptation de [11] et de la Proposition 2.2. Les preuves des Propositions 3.7 et 3.8 sont des conséquences de la Proposition 3.3 et de la Proposition 2.3. De même, les preuves des Propositions 3.9 et 3.10 sont des conséquences de la Proposition 3.5 et de la Proposition 2.3.

\section{Preuve de la Proposition 3.11}

On rappelle que les fonctions $F_{L S}, F_{V 01}, F_{V 02}, F_{H 01}, F_{H 02}, F_{A 01}, F_{A 02}, F_{H 1}, F_{H 2}, F_{A 1}$ et $F_{A 2}$ sont définies par (14).

Pour le jeu de paramètres utilisés dans [11], voir Table A1, ces fonctions vérifient les propriétés suivantes :

$-F_{L S}(D)<F_{H 01}(D)<F_{H 2}(D)<F_{V 01}(D)<F_{A 01}(D)<F_{A 2}(D)$.

$-F_{H 02}(D)<F_{H 01}(D)$.

$-F_{V 01}(D)>F_{V 02}(D)>F_{H 02}(D)$.

$-F_{H 2}(D)>F_{H 1}(D)$.

$-F_{A 1}(D)>F_{A 02}(D), F_{A 1}(D)>F_{V 02}(D)$ et $F_{H 2}(D)>F_{V 02}(D)$.

$-F_{A 2}(D)>F_{A 1}(D)$.

$-F_{V 02}(D)<F_{A 01}(D)$ et $F_{A 02}(D)<F_{A 01}(D)$.

De plus, les fonctions $F_{H 01}(D)$ et $F_{V 02}(D)$ s'intersectent en une valeur $D_{1}>F_{01}$ et vérifient $F_{H 01}(D)<F_{V 02}(D)$ pour $D>D_{1}$, voir figure 15 .

De même, $F_{H 01}(D)$ et $F_{A 02}(D)$ s'intersectent en une valeur $D_{2}>F_{01}$ et vérifient $F_{H 01}(D)<$ $F_{A 02}(D)$ pour $D>D_{2}, F_{H 01}(D)$ et $F_{A 1}(D)$ s'intersectent en une valeur $D_{3}>F_{01}$ et vérifient $F_{H 01}(D)<F_{A 1}(D)$ pour $D>D_{3}, F_{A 1}(D)$ et $F_{H 2}(D)$ s'intersectent en une valeur $D_{4}>F_{01}$ et vérifient $F_{A 1}(D)<F_{H 2}(D)$ pour $D>D_{4}, F_{V 02}(D)$ et $F_{A 02}(D)$ s'intersectent en une valeur $D_{5}>F_{01}$ et vérifient $F_{V 02}(D)>F_{A 02}(D)$ pour $D>D_{5}, F_{V 02}(D)$ et $F_{L S}(D)$ s'intersectent en une valeur $D_{6}>F_{01}$ et vérifient $F_{V 02}(D)>F_{L S}(D)$ pour $D>D_{6}$.

Ce qui définit, les régions $R_{1}, \cdots, R_{11}$, voir figure 12 .

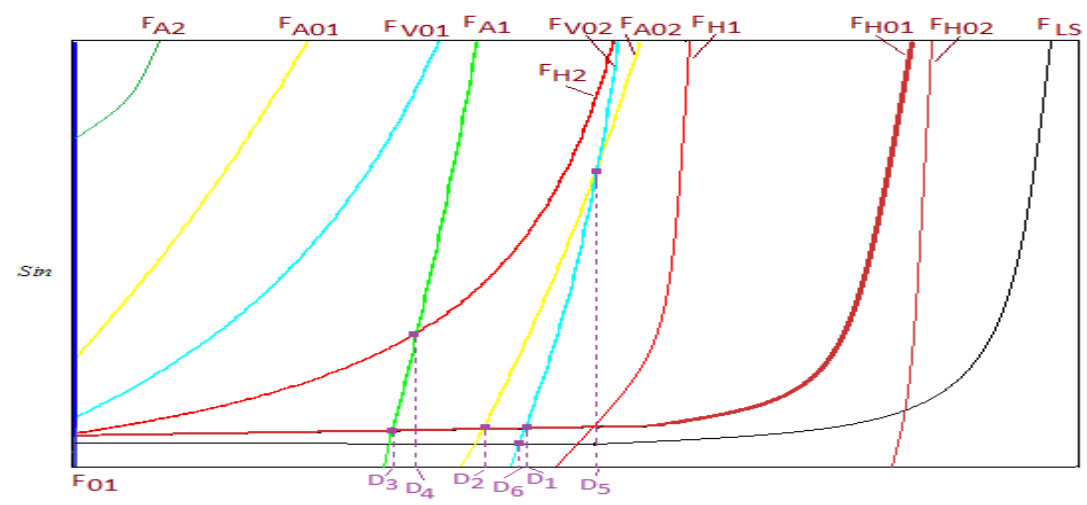

Figure 15. Positions relatives des courbes pour les paramètres utilisés dans [11], Table A1. 


\section{Remerciements}

Les auteurs remercient Tewfik Sari (IRSTEA) pour les discussions fructueuses que nous avons eues à propos de ce travail.

\section{Bibliographie}

[1] T. BAYEn, P. GAJARdo, « On the steady state optimization of the biogas production in a two-stage anaerobic digestion model », J. Math. Biol, vol. 77, 1-21, 2018.

[2] R. Fekih-Salem, N. Abdellatif, T. Sari, J. Harmand, « Analyse mathématique d'un modèle de digestion anaérobie à trois étapes », ARIMA Journal, vol. 17, 53-71,2014.

[3] M. HAJJi, F. MAZENC, J. HARMAND, «A mathematical study of syntrophic relationship of a model of anaerobic digestion process », Math Biosci Eng., vol. 7, 641-656, 2010.

[4] C. Jost, « Predator-Prey Theory : Hidden Twins in Ecology and Microbiology », Oikos, vol. 90(1), 202-208, 2000.

[5] T. SARI, M. El HAJJI, J. HARMAND, « The mathematical analysis of a syntrophic relationship between two microbial species in a chemostat », Math Biosci Eng., vol. 9, 627-645, 2012.

[6] T. SARI, J. HARMAND, «A model of a syntrophic relationship between two microbial species in a chemostat including maintenance », Math Biosci Eng., vol. 275,1-9, 2016.

[7] I. Simeonov, S. StOYAnOv, « Modelling and dynamic compensator control of the anaerobic digestion of organic wastes », Chem. Biochem. Eng. Q, vol. 17(4), 285-292, 2003.

[8] M. Sbarciog, A. VANDE Wouwer, « Bifurcation analysis of an anaerobic digestion system », ICSTCC, 20th International conference on IEEE, 330-335, 2016.

[9] H.L. Smith, P. Waltman, « The Theory of the Chemostat, Dynamics of Microbial Competition », Cambridge University Press, 1995.

[10] V.A. Vavilin, B. Fernandez, J. Palatsi, X. Flotats, « Hydrolysis kinetics in anaerobic degradation of particulate organic material : An overview », Waste Management vol. 28, 939951, 2008.

[11] M. Weedermann, G. Seo, G.S.K.Wolkowicz, « Mathematical model of anaerobic digestion in a chemostat : effects of syntrophy and inhibition », Journal of Biological Dynamics vol. 7(1), 59-85, 2013.

[12] M. WeEdermanN, G.S.K.WolKowICZ, « Optimal biogas production in a model for anaerobic digestion », Nonlinear Dyn. vol. 81, 1097-1112, 2015. 